Advances in Fuzzy Mathematics.

ISSN 0973-533X Volume 12, Number 3 (2017), pp. 439-474

(C) Research India Publications

https://dx.doi.org/10.37622/AFM/12.3.2017.439-474

\title{
Truss Design Optimization with Imprecise Load and Stress in Neutrosophic Environment
}

\author{
Mridula Sarkar* \\ Department of Mathematics, Indian Institute of Engineering Science and Technology, \\ Shibpur, P.O.-Botanic Garden, Howrah-711103, West Bengal, India. \\ Tapan Kumar Roy \\ Department of Mathematics, Indian Institute of Engineering Science and Technology, \\ Shibpur, P.O.-Botanic Garden, Howrah-711103, West Bengal, India. \\ *corresponding author
}

\begin{abstract}
Usually engineering design problems are designed with imprecise parameter in presence of many conflicting objectives . A multi objective three bar truss design problem with imprecise load and stresses has been considered in this paper as test problem. The design variables are cross sectional area of bars. In this problem weight and deflection are considered as objectives and stresses as constraint functions. A neutrosophic mathematical programming has been introduced here to solve such multi-objective structural optimization problem with imprecise parameters. Generalized single valued triangular neutrosophic numbers (GSVNNs) are assumed to be imprecise load and stresses in test problem. In the first step, total integral values of GSVNNs have been calculated for imprecise parameters. Later, a parameterized nonlinear problem has been formulated using ranking method for GSVNNs. Ultimately ,this parameterized multi-objective structural design (MOSD) is solved by using probabilistic operator in neutrosophic environment. The development of such optimization approach also analytically illustrated in this paper. Finally the significance of proposed optimization approach is established by making comparison among results obtained in fuzzy, intuitionistic fuzzy and neutrosophic optimization technique numerically in perspective of structural design.
\end{abstract}

Keywords: Generalized Single Valued Triangular Neutrosophic Number, Total Integral Vaue,Ranking of Generalized Single Valued Neutrosophic Number, Multi-Objective Neutrosophic Optimization, Structural Optimization. 


\section{INTRODUCTION}

Optimization techniques for structural optimal design, consisting of deterministic optimization and non-deterministic optimization methods, have been widely used in practice. The former i.e deterministic optimization aims to search the optimum solution under given constraints without consideration of uncertainties. However, in so many engineering structures, deterministic optimization approaches are unable to handle structural performances exhibit variations such as the fluctuation of external loads, the variation of material properties, e.t.c due to the presence of uncertainties, the so-called optimum solution obtained may lie in the infeasible region. Thus, so many realistic design-approaches must be able to deal with the imprecise nature of structures. This type of optimum solution has been obtained under given reliability constraints, while the later one aims to minimize the variation of the objective function. Such several non-deterministic structural design optimization approaches which are reliability-based design optimization (RBDO), solved by D.M Frangopol et.al[1] and M. Papadrakakis [2], considering structural impreciseness, have been reported in the literature. Moreover in the practical optimization problems usually more than one objective is required to be optimized. Generally they are minimum cost, maximum stiffness ,minimum displacement at specific structural nodes ,maximum natural frequency of free vibration and optimum structural strain energy e.t.c. These make it necessary to formulate a multi-objective optimization problem. The applications of different optimization techniques to structural design, have attracted interest of many researchers. For example Ray Optimization (Kaveh. Et.al [3]), artificial bee colony algorithm (Sonmez, M.[4]),Particle Swarm Optimization (Perez et. al [5],Kaveh et.al [6] and Luh,et.al.[7]),genetic Algorithm (Kaveh,et.al[8],Ali.et.al[9],Dede,et.al[10]), meta heuristic algorithm (Kaveh,A. Motie ,S.Mohammed ,A.,Moslehi,M.[11]),others (Shih, C.j. and Chang,C.J.[12],Hajela,P. and Shih,C.J.[13]Wang ,D.,Zhang,W.H.and Jiang,J.S.[14],Wang ,D.,Zhang,W.H. and Jiang ,J.S.[15];Kripakaran,P.,Gupta,A. and Baugh Jr,J.W.[16]).Fuzzy as well as intuitionistic fuzzy optimization, not only help the engineers, especially in structural engineering, to design and to analyse the systems but also leads to discover fuzzy optimization theory and techniques. This fuzzy set theory was first introduced by Zadeh[17]. As an extension Intuitionistic fuzzy set theory was first introduced by Atanassov[18] .When an imprecise information can not be expressed by means of conventional fuzzy set, intuitionistic fuzzy set plays an important role. In intuitionistic fuzzy (IF) set we usually consider degree of acceptance, and degree of rejection where as we consider only membership function in fuzzy set. A few research work has been done on intuitionistic fuzzy optimization in the field of structural optimization. Dey and Roy [19] used intuitionistic fuzzy technique to optimize single objective two bar truss structural model. A multi-objective intuitionistic optimization technique is applied to optimize three bar truss structural model by Dey and Roy [20] in their paper. When an ill-known information are represented by intuitionistic fuzzy number which is generalization of fuzzy number expresses the available information in flexible way considering non-membership functions. Shu [21] applied triangular intuitionistic fuzzy number to fault tree analysis on printed board circuit assembly.P.Grzegorzewski et.al [22], H.B.Mitchell et.al [23],G.Nayagam et.al 
[24],H.M.Nehi et.al [25],S.Rezvani et.al [26] used concept of intuitionistic fuzzy number in multi-attribute decision making(MADM) problem. Li[27] proposed a ranking method for triangular intuitionistic fuzzy number(TIFN) with definition of ratio of value index to ambiguity index of TIFN in MADM problem as an application. In intuitionistic fuzzy number indeterminate information is partially lost , as hesitant information is taken in consideration by default. So indeterminate information should be considered in decision making process. Smarandache [28]defined neutrosophic set that could handled indeterminate and inconsistent information. In neutrosophic sets indeterminacy is quantified explicitly with truth membership, indeterminacy membership and falsity membership function which are independent .Wang et.al [29] define single valued neutrosophic set which represents imprecise, incomplete, indeterminate, inconsistent information. Thus taking the universe as a real line we can develop the concept of single valued neutrosophic number as special case of neutrosophic sets. These numbers are able to express ill-known quantity with uncertain numerical value in decision making problem. In this present study, we define generalized single valued triangular neutrosophic number and total integral value of this number and using a ranking method of single valued generalized triangular neutrosophic number we solve a multi-objective structural design problem in neutrosophic environment. In this paper we have considered three-bar planer truss subjected to a single load condition. Here the objective functions are weight of the truss and deflection of loaded joint in test problem and the design variables are the cross-sections of bars with the constraints as stresses in members. In this paper we have developed an approach to solve multi-objective structural design using probabilistic operator in neutrosophic environment. Here total integral values of generalized single valued triangular neutrosophic numbers have been considered for applied load and stress.

The remainder of this paper is organized in the following way. In section 2 structural optimization model is discussed. In section 3, mathematics Prerequisites i.e fuzzy set, fuzzy number, single valued neutrosophic set, generalized single valued triangular neutrosophic number (GSVTNN), total integral value of GSVTNN, ranking method of GSVTNN are discussed. In section 4, we have proposed the technique to solve a multi-objective parameterized non-linear programming problem using neutrosophic Optimization technique. In section 5,we have discussed the solution of crisp multi-objective structural model by neutrosophic optimization technique with probabilistic operator. Numerical illustration of structural model of three bar truss are discussed in section 6.Finally we have drawn conclusions in section 7.

\section{MULTI-OBJECTIVE STRUCTURAL MODEL}

A structural design problem may be considered as a minimization type multiobjective nonlinear programming problem where weight and deflection of the loaded joint are to be minimized as objectives and subject to a specified set of stress constraints. The design variables are cross sectional area of bars . The target of optimization is the identification of the optimum cross-sectional area of bar so that the 
structure can achieve its smallest total weight with minimum nodal displacement in a given load conditions .

The multi-objective structural model can be expressed as

Minimize $W T(A)$

Minimize $\delta(A)$

subject to $\sigma_{i}(A) \leq\left[\sigma_{i}\right]$

$A^{\min } \leq A \leq A^{\max }$

Where $A=\left[A_{1}, A_{2}, \ldots A_{n}\right]^{T}$ are the design variables for the cross section, $\mathrm{n}$ is the group number of design variables for the cross section of bars, $W T(A)=\sum_{i=1}^{n} \rho_{i} A_{i} L_{i}$ is the total weight of the structure, $\delta(A)$ is the deflection of the loaded joint, where $L_{i}, A_{i}$ and $\rho_{i}$ are the length of bar, cross section area and density of the $i^{\text {th }}$ group bars respectively. $\sigma_{i}(A)$ is the stress constraints and $\left[\sigma_{i}\right]$ is allowable stress of the group bars under various conditions, $A^{\mathrm{min}}$ and $A^{\mathrm{max}}$ are the lower and upper bounds of cross section area A respectively.

\section{MATHEMATICAL PRELIMINARIES}

\subsection{Fuzzy Set}

A fuzzy set $\tilde{A}$ in a universe of discourse $X$ is defined by $\tilde{A}=\left\{\left(x, \mu_{\tilde{A}}(x)\right) \mid x \in X\right\}$ where $\mu_{\tilde{A}}(x): X \rightarrow[0,1]$ is called the membership function of $\tilde{A}$ and the value of $\mu_{\tilde{A}}(x)$ is called the degree of membership for $x \in X$.

The $\alpha$-cut of fuzzy set A is the crisp set $A_{\alpha}$ given by $A_{\alpha}=\left\{x \in X: \mu_{A}(x) \geq \alpha\right\} \alpha \in[0,1]$

\subsection{Generalized Fuzzy Number}

A fuzzy set $\tilde{A}$ of the real line $\square$ with membership function $\mu_{\tilde{A}}(x): \square \rightarrow[0, w] w \in[0,1]$ is called a generalized fuzzy number if

1. $\tilde{A}$ is normal ,i.e there exists an element $x_{0} \in \square$ such that $\mu_{\tilde{A}}\left(x_{0}\right)=w$ where $w \in[0,1]$.

2. $\tilde{A}$ is convex ,i.e $\mu_{\tilde{A}}\left\{\lambda x_{1}+(1-\lambda) x_{2}\right\} \geq \min \left\{\mu_{\tilde{A}}\left(x_{1}\right), \mu_{\tilde{A}}\left(x_{2}\right)\right\}$ for all $x_{1}, x_{2} \in \square$ and $\lambda \in[0,1]$.

3. $\mu_{\tilde{\AA}}$ is upper semi-continuous, and

4.The support of $\tilde{A}$ i.e $S(\tilde{A})=\left\{x \in X: \mu_{\tilde{A}}(x)>0\right\}$ is bounded. 


\subsection{Triangular Fuzzy Number}

A fuzzy number $\tilde{A}$ is called triangular fuzzy number (TFN) ,if its membership function is defined by $\mu_{\tilde{A}}(x)=\left\{\begin{array}{c}w_{a}\left(\frac{x-a_{1}^{\mu}}{a_{2}-a_{1}^{\mu}}\right) \text { if } a_{1}^{\mu} \leq x \leq a_{2} \\ w_{a}\left(\frac{a_{3}^{\mu}-x}{a_{3}^{\mu}-a_{2}}\right) \text { if } a_{2} \leq x \leq a_{3}^{\mu} \\ 0 \text { otherwise }\end{array}\right.$

The generalized TFN is denoted by $\tilde{A}=\left(a_{1}^{\mu}, a_{2}, a_{3}^{\mu} ; w_{a}\right)$ where $a_{1}^{\mu}, a_{2}, a_{3}^{\mu}$ are real numbers and $a_{1}^{\mu} \leq a_{2} \leq a_{3}^{\mu}$ and $w_{a} \in[0,1]$. The value of $x$ at $a_{2}$ gives the maximum value of $\mu_{\tilde{A}}(x)$ i.e $\mu_{\tilde{A}}(x)=w_{a}$.It is the most probable value of evaluation data.The value of $x$ outside the interval $\left[a_{1}^{\mu}, a_{3}^{\mu}\right]$ gives the minimum value of $\mu_{\tilde{A}}(x)$ i.e $\mu_{\tilde{A}}(x)=0$. It is the least probable value of the evaluation data. constants $a_{1}^{\mu}, a_{3}^{\mu}$ are the lower and upper bounds of the available area for the evaluation data.

The $\alpha-$ cut of generalized TFN $\left(a_{1}^{\mu}, a_{2}, a_{3}^{\mu} ; w_{a}\right)$ is the closed interval $A_{\alpha}=\left[L^{\alpha}(A), R^{\alpha}(A)\right]=\left[a_{1}^{\mu}+\frac{\alpha}{w_{a}}\left(a_{2}-a_{1}^{\mu}\right), a_{3}^{\mu}-\frac{\alpha}{w_{a}}\left(a_{3}^{\mu}-a_{2}\right)\right]$.

\subsection{Single Valued Neutrosophic Set}

A single valued neutrosophic set (SVNS) $\tilde{A}^{n}$ in the universe of discourse $X$ is given $\tilde{A}^{n}=\left\{\left(x, T_{A^{n}}(x), I_{A^{n}}(x), F_{A^{n}}(x)\right) \mid x \in X\right\}$ where $\quad T_{A^{n}}: X \rightarrow[0,1], I_{A^{n}}: X \rightarrow[0,1]$ and $F_{A^{n}}: X \rightarrow[0,1]$ with $0 \leq T_{A^{n}}(x)+I_{A^{n}}(x)+F_{A^{n}}(x) \leq 3$ for all $x \in X$. The numbers $T_{A^{n}}(x), I_{A^{n}}(x), F_{A^{n}}(x)$, respectively represent the truth membership degree, indeterminacy membership degree, falsity membership degree of the element $x$ to the set $\tilde{A}^{n}$.

\section{5. $(\alpha, \beta, \gamma)$ - cut of Single Valued Neutrosophic Set}

$(\alpha, \beta, \gamma)$-cut of single valued neutrosophic set (SVNS) $\tilde{A}^{n}$, a crisp subset of $\square$ is defined by $A_{\alpha, \beta, \gamma}^{n}=\left\{x \mid T_{A^{n}}(x) \geq \alpha, I_{A^{n}}(x) \leq \beta, F_{A^{n}}(x) \leq \gamma\right\}$ where $\alpha, \beta, \gamma \in[0,1]$ and $0 \leq \alpha+\beta+\gamma \leq 3$. 


\subsection{Normal Neutrosophic Set}

A single valued neutrosophic set $\tilde{A}^{n}=\left\{\left(x, T_{A^{n}}(x), I_{A^{n}}(x), F_{A^{n}}(x)\right) \mid x \in X\right\}$ is called neutrosophic normal if there exists at least three points $x_{0}, x_{1}, x_{2} \in X$ such that $T_{A^{n}}\left(x_{0}\right)=1$

$I_{A^{n}}\left(x_{1}\right)=1, I_{A^{n}}\left(x_{2}\right)=1$.

\subsection{Convex Neutrosophic Set}

A single valued neutrosophic set $\tilde{A}^{n}=\left\{\left(x, T_{A^{n}}(x), I_{A^{n}}(x), F_{A^{n}}(x)\right) \mid x \in X\right\}$ is a subset of the real line called neut-convex if for all $x_{1}, x_{2} \in \square$ and $\lambda \in[0,1]$ the following conditions are satisfied.

1. $T_{A^{n}}\left\{\lambda x_{1}+(1-\lambda) x_{2}\right\} \geq \min \left\{T_{A^{n}}\left(x_{1}\right), T_{A^{n}}\left(x_{2}\right)\right\}$

2. $I_{A^{n}}\left\{\lambda x_{1}+(1-\lambda) x_{2}\right\} \leq \max \left\{I_{A^{n}}\left(x_{1}\right), I_{A^{n}}\left(x_{2}\right)\right\}$

3. $F_{A^{n}}\left\{\lambda x_{1}+(1-\lambda) x_{2}\right\} \leq \max \left\{F_{A^{n}}\left(x_{1}\right), F_{A^{n}}\left(x_{2}\right)\right\}$

i.e $\tilde{A}^{n}$ is neut-convex if its truth membership function is fuzzy convex, indeterminacy membership function is fuzzy concave and falsity membership function is fuzzy concave.

\subsection{Single Valued Neutrosophic Number}

A single valued neutrosophic set $\tilde{A}^{n}=\left\{\left(x, T_{A^{n}}(x), I_{A^{n}}(x), F_{A^{n}}(x)\right) \mid x \in X\right\}$, subset of a real line, is called generalised neutrosophic number if

1. $\tilde{A}^{n}$ is neut- normal.

2. $\tilde{A}^{n}$ is neut- convex.

3. $T_{A^{n}}(x)$ is upper semi-continuous, $I_{A^{n}}(x)$ is lower semi continuous and $F_{A^{n}}(x)$ is lower semi continuous , and

4. the support of $\tilde{A}^{n}$,i.e. $S\left(\tilde{A}^{n}\right)=\left\{x \in X: T_{A^{n}}>0, I_{A^{n}}<1, F_{A^{n}}<1\right\}$ is bounded.

Thus for any Single Valued Triangular Neutrosophic Number there exists nine numbers $a_{1}^{T}, a_{2}, a_{3}^{T}, b_{1}^{I}, b_{2}, b_{3}^{I}, c_{1}^{F}, c_{2}, c_{3}^{F} \in \square$ such that $c_{1}^{F} \leq b_{1}^{I} \leq a_{1}^{T} \leq c_{2} \leq b_{2} \leq a_{2} \leq a_{3}^{T} \leq b_{3}^{I} \leq c_{3}^{F}$ and six functions $\quad T_{\tilde{A}^{n}}^{L}(x), I_{\tilde{A}^{n}}^{L}(x), F_{\tilde{A}^{n}}^{L}(x), T_{\tilde{A}^{n}}^{R}(x), I_{\tilde{A}^{n}}^{R}(x), F_{\tilde{A}^{n}}^{R}(x): \square \rightarrow[0,1]$ represents truth, indeterminacy and falsity membership degree of $\tilde{A}^{n}$.The three non-decreasing functions $T_{\tilde{A}^{n}}^{L}(x), I_{\tilde{A}^{n}}^{L}(x), F_{\tilde{A}^{n}}^{L}(x)$ represents the left side of truth, indeterminacy and falsity membership functions of SVNN $\tilde{A}^{n}$ respectively. Similarly the three nonincreasing functions $T_{\tilde{A}^{n}}^{R}(x), I_{\tilde{A}^{n}}^{R}(x), F_{\tilde{A}^{n}}^{R}(x)$ represent the right side of truth, indeterminacy and falsity membership functions of SVNN $\tilde{A}^{n}$ respectively. The truth, 
indeterminacy and falsity membership functions of SVNN $\tilde{A}^{n}$ can be defined in the following way

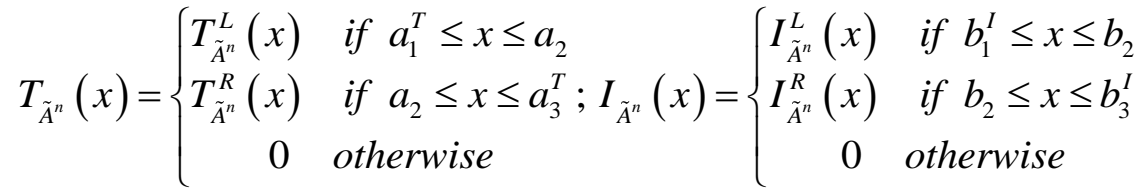

$$
\begin{aligned}
& F_{\tilde{A}^{n}}(x)=\left\{\begin{array}{c}
F_{\tilde{A}^{n}}^{L}(x) \text { if } c_{1}^{F} \leq x \leq c_{2} \\
F_{\tilde{A}^{n}}^{R}(x) \text { if } c_{2} \leq x \leq c_{3}^{F} \\
0 \quad \text { otherwise }
\end{array}\right.
\end{aligned}
$$

The sum of three independent membership degree of SVNN $\tilde{A}^{n}$ lie between the interval $[0,3]$.i.e $0 \leq T_{\tilde{A}^{n}}^{R}(x)+I_{\tilde{A}^{n}}^{R}(x)+F_{\tilde{A}^{n}}^{R}(x) \leq 3 \quad x \in \tilde{A}^{n}$.

\subsection{Generalized Triangular Neutrosophic Number}

A generalized single valued triangular Neutrosophic number $\tilde{A}^{n}$ with the set of parameters $c_{1}^{F} \leq b_{1}^{I} \leq a_{1}^{T} \leq c_{2} \leq b_{2} \leq a_{2} \leq a_{3}^{T} \leq b_{3}^{I} \leq c_{3}^{F}$ denoted as $\tilde{A}^{n}=\left(\left(a_{1}^{T}, a_{2}, a_{3}^{T} ; w_{a}\right),\left(b_{1}^{I}, b_{2}, b_{3}^{I} ; \eta_{a}\right)\left(c_{1}^{F}, c_{2}, c_{3}^{F} ; \tau_{a}\right)\right)$ is the set of real numbers $\square$.The truth membership, indeterminacy membership and falsity membership functions of $\tilde{A}^{n}$ can be defined as follows

$$
\begin{aligned}
& T_{\tilde{A}^{n}}=\left\{\begin{array}{cl}
w_{a} \frac{x-a_{1}^{T}}{a_{2}-a_{1}^{T}} & \text { for } a_{1}^{T} \leq x \leq a_{2} \\
w_{a} \quad \text { for } x=a_{2} \\
w_{a} \frac{a_{3}^{T}-x}{a_{3}^{T}-a_{2}} \text { for } a_{2} \leq x \leq a_{3}^{T} \\
0 \quad \text { otherwise }
\end{array}\right. \\
& I_{\tilde{A}^{n}}=\left\{\begin{array}{cl}
\eta_{a} \frac{x-b_{1}^{I}}{b_{2}-b_{1}^{I}} & \text { for } b_{1}^{I} \leq x \leq b_{2} \\
\eta_{a} & \text { for } x=b_{2} \\
\eta_{a} \frac{x-b_{3}^{I}}{b_{3}^{I}-b_{2}} & \text { for } b_{2} \leq x \leq b_{3}^{I} \\
0 \quad \text { otherwise }
\end{array}\right. \\
& F_{\tilde{A}^{n}}=\left\{\begin{array}{cl}
\tau_{a} \frac{x-c_{1}^{F}}{c_{2}-c_{1}^{F}} & \text { for } c_{1}^{F} \leq x \leq c_{2} \\
\tau_{a} & \text { for } x=c_{2} \\
\tau_{a} \frac{x-c_{3}^{F}}{c_{3}^{F}-c_{2}} & \text { for } c_{2} \leq x \leq c_{3}^{F} \\
0 \quad \text { otherwise }
\end{array}\right.
\end{aligned}
$$




\subsection{0. $(\alpha, \beta, \gamma)$-cut Set of Single Valued Triangular Neutrosophic Number}

Let $\tilde{A}^{n}=\left(\left(a_{1}^{T}, a_{2}, a_{3}^{T} ; w_{a}\right),\left(b_{1}^{I}, b_{2}, b_{3}^{I} ; \eta_{a}\right)\left(c_{1}^{F}, c_{2}, c_{3}^{F} ; \tau_{a}\right)\right)$ be generalized single valued triangular Neutrosophic number. Then it is a crisp subset of $\square$ and is defined by

$$
\begin{aligned}
A_{\alpha, \beta, \gamma}^{n}= & \left\{x \mid T_{A^{n}}(x) \geq \alpha, I_{A^{n}}(x) \leq \beta, F_{A^{n}}(x) \leq \gamma\right\} \\
& =\left\{\left[L^{\alpha}(\tilde{A}), R^{\alpha}(\tilde{A})\right],\left[L^{\beta}(\tilde{A}), R^{\beta}(\tilde{A})\right],\left[L^{\gamma}(\tilde{A}), R^{\gamma}(\tilde{A})\right]\right\} \\
& =\left\{\begin{array}{l}
{\left[a_{1}^{T}+\frac{\alpha}{w_{a}}\left(a_{2}-a_{1}^{T}\right), a_{3}^{T}-\frac{\alpha}{w_{a}}\left(a_{3}^{T}-a_{2}\right)\right],} \\
{\left[b_{1}^{I}+\frac{\beta}{\eta_{a}}\left(b_{2}-b_{1}^{I}\right), b_{3}^{I}+\frac{\beta}{\eta_{a}}\left(b_{3}^{I}-b_{2}\right)\right],} \\
{\left[c_{1}^{F}+\frac{\gamma}{\tau_{a}}\left(c_{2}-c_{1}^{F}\right), c_{3}^{F}+\frac{\gamma}{\tau_{a}}\left(c_{3}^{F}-c_{2}\right)\right]}
\end{array}\right\}
\end{aligned}
$$

\subsection{Property}

\section{Property:3.11.1.}

Let $\tilde{A}^{n}=\left(\left(a_{1}^{T}, a_{2}, a_{3}^{T} ; w_{a}\right),\left(b_{1}^{I}, b_{2}, b_{3}^{I} ; \eta_{a}\right)\left(c_{1}^{F}, c_{2}, c_{3}^{F} ; \tau_{a}\right)\right)$ and $\tilde{B}^{n}=\left(\left(e_{1}^{T}, e_{2}, e_{3}^{T} ; w_{b}\right),\left(f_{1}^{I}, f_{2}, f_{3}^{I} ; \eta_{b}\right)\left(g_{1}^{F}, g_{2}, g_{3}^{F} ; \tau_{b}\right)\right)$ be two generalized triangular neutrosophic number then the arithmetic operations on these numbers can be defined as follows

$$
\tilde{A}^{n}+\tilde{B}^{n}=\left(\begin{array}{c}
\left(a_{1}^{T}+e_{1}^{T}, a_{2}+e_{2}, a_{3}^{T}+e_{3}^{T} ; \min \left(w_{a}, w_{b}\right)\right) \\
\left(b_{1}^{I}+f_{1}^{I}, b_{2}+f_{2}, b_{3}^{I}+f_{3}^{I} ; \max \left(\eta_{a}, \eta_{b}\right)\right) \\
\left(c_{1}^{F}+g_{1}^{F}, c_{2}+g_{2}, c_{3}^{F}+g_{3}^{F} ; \max \left(\tau_{a}, \tau_{b}\right)\right)
\end{array}\right)
$$

\section{Proof:}

With the transformation $z=x+y$ we can find truth, indeterminacy and falsity membership function of neutrosophic set $\tilde{C}^{n}=\tilde{A}^{n}+\tilde{B}^{n}$ by $\alpha, \beta, \gamma$-cut method. $\alpha-$ cut of $\tilde{A}^{n}$ is $\left[a_{1}^{T}+\frac{\alpha}{w_{a}}\left(a_{2}-a_{1}^{T}\right), a_{3}^{T}-\frac{\alpha}{w_{a}}\left(a_{3}^{T}-a_{2}\right)\right] \forall \alpha \in[0,1]$ i.e $x \in\left[a_{1}^{T}+\frac{\alpha}{w_{a}}\left(a_{2}-a_{1}^{T}\right), a_{3}^{T}-\frac{\alpha}{w_{a}}\left(a_{3}^{T}-a_{2}\right)\right] . \alpha-$ cut of $\tilde{B}^{n}$ is $\left[e_{1}^{T}+\frac{\alpha}{w_{b}}\left(e_{2}-e_{1}^{T}\right), e_{3}^{T}-\frac{\alpha}{w_{b}}\left(e_{3}^{T}-e_{2}\right)\right] \forall \alpha \in[0,1]$ i.e 
Truss Design Optimization with Imprecise Load and Stress in Neutrosophic En-vironment 447

$$
\begin{aligned}
& y \in\left[e_{1}^{T}+\frac{\alpha}{w_{b}}\left(e_{2}-e_{1}^{T}\right), e_{3}^{T}-\frac{\alpha}{w_{b}}\left(e_{3}^{T}-e_{2}\right)\right] \text { So } \\
& z(=x+y) \in\left[a_{1}^{T}+e_{1}^{T}+\frac{\alpha}{w}\left(\left(e_{2}-e_{1}^{T}\right)+\left(a_{2}-a_{1}^{T}\right)\right), a_{3}^{T}+e_{3}^{T}-\frac{\alpha}{w}\left(\left(e_{3}^{T}-e_{2}\right)+\left(a_{3}^{T}-a_{2}\right)\right)\right]
\end{aligned}
$$

Where $w=\min \left\{w_{a}, w_{b}\right\}$

Thus we get truth membership function of $\tilde{C}^{n}=\tilde{A}^{n}+\tilde{B}^{n}$ as

$$
T_{\tilde{C}^{n}}(z)=\left\{\begin{array}{cl}
w\left(\frac{z-a_{1}^{T}-e_{1}^{T}}{\left(a_{2}-a_{1}^{T}\right)+\left(e_{2}-e_{1}^{T}\right)}\right) & \text { for } a_{1}^{T}+e_{1}^{T} \leq z \leq a_{2}+e_{2} \\
w\left(\frac{a_{3}^{T}+e_{3}^{T}-z}{\left(a_{3}^{T}-a_{2}\right)+\left(e_{3}^{T}-e_{2}\right)}\right) & \text { for } a_{2}+e_{2} \leq z \leq a_{3}^{T}+e_{3}^{T} \\
0 & \text { otherwize }
\end{array}\right.
$$

Hence the rule is proved for truth membership function.

$$
\begin{aligned}
& \beta \text {-cut of } \tilde{A}^{n} \text { is }\left[b_{1}^{I}+\frac{\beta}{\eta_{a}}\left(b_{2}-b_{1}^{I}\right), b_{3}^{I}+\frac{\beta}{\eta_{a}}\left(b_{3}^{I}-b_{2}\right)\right] \forall \beta \in[0,1] \text { i.e } \\
& x \in\left[b_{1}^{I}+\frac{\beta}{\eta_{a}}\left(b_{2}-b_{1}^{I}\right), b_{3}^{I}+\frac{\beta}{\eta_{a}}\left(b_{3}^{I}-b_{2}\right)\right] \cdot \beta-\text { cut of } \tilde{B}^{n} \text { is } \\
& {\left[f_{1}^{I}+\frac{\beta}{\eta_{b}}\left(f_{2}-f_{1}^{I}\right), f_{3}^{I}+\frac{\beta}{\eta_{b}}\left(f_{3}^{I}-f_{2}\right)\right] \forall \beta \in[0,1] \text { i.e }} \\
& y \in\left[f_{1}^{I}+\frac{\beta}{\eta_{b}}\left(f_{2}-f_{1}^{I}\right), f_{3}^{I}+\frac{\beta}{\eta_{b}}\left(f_{3}^{I}-f_{2}\right)\right] . \text { So } \\
& z(=x+y) \in\left[b_{1}^{I}+f_{1}^{I}+\frac{\beta}{\eta}\left(\left(b_{2}-b_{1}^{I}\right)+\left(f_{2}-f_{1}^{I}\right)\right), b_{3}^{I}+f_{3}^{I}+\frac{\beta}{\eta}\left(\left(b_{3}^{I}-b_{2}\right)+\left(f_{3}^{I}-f_{2}\right)\right)\right]
\end{aligned}
$$

Where $\eta=\max \left\{\eta_{a}, \eta_{b}\right\}$

Thus we get indeterminacy membership function of $\tilde{C}^{n}=\tilde{A}^{n}+\tilde{B}^{n}$ as 
$I_{\tilde{C}^{n}}(z)=\left\{\begin{array}{cl}\eta\left(\frac{b_{1}^{I}+f_{1}^{I}-z}{\left(b_{2}-b_{1}^{I}\right)+\left(f_{2}-f_{1}^{I}\right)}\right) & \text { for } b_{1}^{I}+f_{1}^{I} \leq z \leq b_{2}+f_{2} \\ \eta\left(\frac{z-b_{3}^{I}-f_{3}^{I}}{\left(b_{3}^{I}-b_{2}\right)+\left(f_{3}^{I}-f_{2}\right)}\right) & \text { for } b_{2}+f_{2} \leq z \leq b_{3}^{I}+f_{3}^{I} \\ 0 & \text { otherwize }\end{array}\right.$

Hence the rule is proved for indeterminacy membership function.

$$
\begin{aligned}
& \gamma-\text { cut of } \tilde{A}^{n} \text { is }\left[c_{1}^{F}+\frac{\gamma}{\tau_{a}}\left(c_{2}-c_{1}^{F}\right), c_{3}^{F}+\frac{\gamma}{\tau_{a}}\left(c_{3}^{F}-c_{2}\right)\right] \forall \gamma \in[0,1] \text { i.e } \\
& x \in\left[c_{1}^{F}+\frac{\gamma}{\tau_{a}}\left(c_{2}-c_{1}^{F}\right), c_{3}^{F}+\frac{\gamma}{\tau_{a}}\left(c_{3}^{F}-c_{2}\right)\right] \cdot \gamma-\text { cut of } \tilde{B}^{n} \text { is } \\
& {\left[g_{1}^{F}+\frac{\gamma}{\tau_{b}}\left(g_{2}-g_{1}^{F}\right), g_{3}^{F}+\frac{\gamma}{\tau_{b}}\left(g_{3}^{F}-g_{2}\right)\right] \forall \gamma \in[0,1] \text { i.e }} \\
& y \in\left[g_{1}^{F}+\frac{\gamma}{\tau_{b}}\left(g_{2}-g_{1}^{F}\right), g_{3}^{F}+\frac{\gamma}{\tau_{b}}\left(g_{3}^{F}-g_{2}\right)\right] \cdot \text { So } \\
& z(=x+y) \in\left[c_{1}^{F}+g_{1}^{F}+\frac{\gamma}{\tau}\left(\left(c_{2}-c_{1}^{F}\right)+\left(g_{2}-g_{1}^{F}\right)\right), c_{3}^{F}+g_{3}^{F}+\frac{\gamma}{\tau}\left(\left(c_{3}^{F}-c_{2}\right)+\left(g_{3}^{F}-g_{2}\right)\right)\right]
\end{aligned}
$$

Where $\tau=\max \left\{\tau_{a}, \tau_{b}\right\}$

Thus we get falsity membership function of $\tilde{C}^{n}=\tilde{A}^{n}+\tilde{B}^{n}$ as

$$
F_{\tilde{C}^{n}}(z)=\left\{\begin{array}{cl}
\tau\left(\frac{c_{1}^{F}+g_{1}^{F}-z}{\left(c_{2}-c_{1}^{F}\right)+\left(g_{2}-g_{1}^{F}\right)}\right) & \text { for } c_{1}^{F}+g_{1}^{F} \leq z \leq c_{2}+g_{2} \\
\tau\left(\frac{z-c_{3}^{F}-g_{3}^{F}}{\left(c_{3}^{F}-c_{2}\right)+\left(g_{3}^{F}-g_{2}\right)}\right) & \text { for } c_{2}+g_{2} \leq z \leq c_{3}^{F}+g_{3}^{F} \\
0 & \text { otherwize }
\end{array}\right.
$$

Hence the rule is proved for falsity membership function.

Hence the proof 
Truss Design Optimization with Imprecise Load and Stress in Neutrosophic En-vironment 449

\section{Property:3.11.2.}

Let $\tilde{A}^{n}=\left(\left(a_{1}^{T}, a_{2}, a_{3}^{T} ; w_{a}\right),\left(b_{1}^{I}, b_{2}, b_{3}^{I} ; \eta_{a}\right)\left(c_{1}^{F}, c_{2}, c_{3}^{F} ; \tau_{a}\right)\right)$ be a generalized triangular neutrosophic number then

$$
k \tilde{A}^{n}=\left\{\begin{array}{l}
\left(k a_{1}^{T}, k a_{2}, k a_{3}^{T} ; w_{a}\right),\left(k b_{1}^{I}, k b_{2}, k b_{3}^{I} ; \eta_{a}\right)\left(k c_{1}^{F}, k c_{2}, k c_{3}^{F} ; \tau_{a}\right) \text { for } k>0 \\
\left(k a_{3}^{T}, k a_{2}, k a_{1}^{T} ; w_{a}\right),\left(k b_{3}^{I}, k b_{2}, k b_{1}^{I} ; \eta_{a}\right)\left(k c_{3}^{F}, k c_{2}, k c_{1}^{F} ; \tau_{a}\right) \text { for } k<0
\end{array}\right.
$$

\section{Proof:}

When $k>0$, with transformation $y=k a$ we can find the membership function for truth membership function of TNN $\tilde{Y}^{n}=k \tilde{A}^{n}$ by $\alpha$-cut method. The $\alpha$-cut of $\tilde{A}^{n}$ is $\left[a_{1}^{T}+\frac{\alpha}{w_{a}}\left(a_{2}-a_{1}^{T}\right), a_{3}^{T}+\frac{\alpha}{w_{a}}\left(a_{3}^{T}-a_{2}\right)\right]$ i.e $x \in\left[a_{1}^{T}+\frac{\alpha}{w_{a}}\left(a_{2}-a_{1}^{T}\right), a_{3}^{T}+\frac{\alpha}{w_{a}}\left(a_{3}^{T}-a_{2}\right)\right]$

So $y(=k a) \in\left[k a_{1}^{T}+\frac{\alpha}{w_{a}}\left(k a_{2}-k a_{1}^{T}\right), k a_{3}^{T}+\frac{\alpha}{w_{a}}\left(k a_{3}^{T}-k a_{2}\right)\right]$

Thus we get truth membership function of $\tilde{Y}^{n}=k \tilde{A}^{n}$ as

$$
T_{\tilde{Y}^{n}}(y)=\left\{\begin{array}{cc}
w_{a}\left(\frac{y-k a_{1}^{T}}{\left(k a_{2}-k a_{1}^{T}\right)}\right) & \text { for } k a_{1}^{T} \leq y \leq k a_{2} \\
w_{a}\left(\frac{k a_{3}^{T}-y}{\left(k a_{3}^{T}-k a_{2}\right)}\right) & \text { for } k a_{2} \leq y \leq k a_{1}^{T} \\
0 & \text { otherwise }
\end{array}\right.
$$

Hence the rule is proved for truth membership function .

The $\beta-$ cut of $\tilde{A}^{n} \quad$ is $\left[b_{1}^{I}+\frac{\beta}{\eta_{a}}\left(b_{2}-b_{1}^{I}\right), b_{3}^{I}+\frac{\beta}{\eta_{a}}\left(b_{3}^{I}-b_{2}\right)\right] \forall \beta \in[0,1] \quad$ i.e $x \in\left[b_{1}^{I}+\frac{\beta}{\eta_{a}}\left(b_{2}-b_{1}^{I}\right), b_{3}^{I}+\frac{\beta}{\eta_{a}}\left(b_{3}^{I}-b_{2}\right)\right] \forall \beta \in[0,1]$

So $y(=k a) \in\left[k b_{1}^{I}+\frac{\beta}{\eta_{a}}\left(k b_{2}-k b_{1}^{I}\right), k b_{3}^{I}+\frac{\beta}{\eta_{a}}\left(k b_{3}^{I}-k b_{2}\right)\right] \forall \beta \in[0,1]$ Thus we get indeterminacy membership function of $\tilde{Y}^{n}=k \tilde{A}^{n}$ as 


$$
I_{\tilde{Y}^{n}}(y)=\left\{\begin{array}{cc}
\eta_{a}\left(\frac{k b_{1}^{I}-y}{\left(k b_{2}-k b_{1}^{I}\right)}\right) & \text { for } k b_{1}^{I} \leq y \leq k b_{2} \\
\eta_{a}\left(\frac{y-k b_{3}^{I}}{\left(k b_{3}^{I}-k b_{2}\right)}\right) & \text { for } k b_{2} \leq y \leq k b_{3}^{I} \\
0 & \text { otherwise }
\end{array}\right.
$$

Hence the rule is proved for indeterminacy-membership function .

The $\gamma-$ cut of $\tilde{A}^{n}$ is $\left[\left[c_{1}^{F}+\frac{\gamma}{\tau_{a}}\left(c_{2}-c_{1}^{F}\right), c_{3}^{F}+\frac{\gamma}{\tau_{a}}\left(c_{3}^{F}-c_{2}\right)\right]\right] \forall \gamma \in[0,1] \quad$ i.e $x \in\left[\left[c_{1}^{F}+\frac{\gamma}{\tau_{a}}\left(c_{2}-c_{1}^{F}\right), c_{3}^{F}+\frac{\gamma}{\tau_{a}}\left(c_{3}^{F}-c_{2}\right)\right]\right] \forall \gamma \in[0,1]$

So $y(=k a) \in\left[\left[k c_{1}^{F}+\frac{\gamma}{\tau_{a}}\left(k c_{2}-k c_{1}^{F}\right), k c_{3}^{F}+\frac{\gamma}{\tau_{a}}\left(k c_{3}^{F}-k c_{2}\right)\right]\right] \forall \gamma \in[0,1]$ Thus we get falsity membership function of $\tilde{Y}^{n}=k \tilde{A}^{n}$ as

$$
F_{\tilde{Y}^{n}}(y)=\left\{\begin{array}{cl}
\tau_{a}\left(\frac{k c_{1}^{F}-y}{\left(k c_{2}-k c_{1}^{I}\right)}\right) & \text { for } k c_{1}^{F} \leq y \leq k c_{2} \\
\tau_{a}\left(\frac{y-k c_{3}^{F}}{\left(k c_{3}^{F}-k c_{2}\right)}\right) & \text { for } k c_{2} \leq y \leq k c_{3}^{F} \\
0 & \text { otherwise }
\end{array}\right.
$$

Hence the rule is proved for falsity-membership function .

Thus we have

$$
k \tilde{A}^{n}=\left\{\begin{array}{l}
\left(k a_{1}^{T}, k a_{2}, k a_{3}^{T} ; w_{a}\right),\left(k b_{1}^{I}, k b_{2}, k b_{3}^{I} ; \eta_{a}\right)\left(k c_{1}^{F}, k c_{2}, k c_{3}^{F} ; \tau_{a}\right) \text { for } k>0 \\
\left(k a_{3}^{T}, k a_{2}, k a_{1}^{T} ; w_{a}\right),\left(k b_{3}^{I}, k b_{2}, k b_{1}^{I} ; \eta_{a}\right)\left(k c_{3}^{F}, k c_{2}, k c_{1}^{F} ; \tau_{a}\right) \text { for } k<0
\end{array}\right.
$$

\subsection{Ranking of Triangular Neutrosophic Number}

A triangular neutrosophic number $\tilde{A}^{n}=\left(\left(a_{1}^{T}, a_{2}, a_{3}^{T} ; w_{a}\right),\left(b_{1}^{I}, b_{2}, b_{3}^{I} ; \eta_{a}\right)\left(c_{1}^{F}, c_{2}, c_{3}^{F} ; \tau_{a}\right)\right)$ is completely defined by $L_{T}(x)=w_{a} \frac{x-a_{1}^{T}}{a_{2}-a_{1}^{T}}$ for $a_{1}^{T} \leq x \leq a_{2}$ and 
$R_{T}(x)=w_{a} \frac{a_{3}^{T}-x}{a_{3}^{T}-a_{2}}$ for $a_{2} \leq x \leq a_{3}^{T} ; L_{I}(x)=\eta_{a} \frac{x-b_{1}^{I}}{b_{2}-b_{1}^{I}}$ for $b_{1}^{I} \leq x \leq b_{2}$ and

$R_{I}(x)=\eta_{a} \frac{x-b_{3}^{I}}{b_{3}^{I}-b_{2}}$ for $b_{2} \leq x \leq b_{3}^{I}$;

$L_{F}(x)=\tau_{a} \frac{x-c_{1}^{F}}{c_{2}-c_{1}^{F}}$ for $c_{1}^{F} \leq x \leq c_{2}$ and $R_{F}(x)=\tau_{a} \frac{x-c_{3}^{F}}{c_{3}^{F}-c_{2}}$ for $c_{2} \leq x \leq c_{3}^{F}$. The

inverse The inverse functions can be analytically expressed as

$L_{T}^{-1}(h)=a_{1}^{T}+\frac{h}{w_{a}}\left(a_{2}-a_{1}^{T}\right) ; R_{T}^{-1}(h)=a_{3}^{T}-\frac{h}{w_{a}}\left(a_{3}^{T}-a_{2}\right) ; L_{I}^{-1}(h)=b_{1}^{I}+\frac{h}{\eta_{a}}\left(b_{2}-b_{1}^{I}\right) ;$

$R_{I}^{-1}(h)=b_{3}^{I}+\frac{h}{\eta_{a}}\left(b_{3}^{I}-b_{2}\right)$

$L_{F}^{-1}(h)=c_{1}^{F}+\frac{h}{\tau_{a}}\left(c_{2}-c_{1}^{F}\right)$ and $R_{F}^{-1}(h)=c_{3}^{F}+\frac{h}{\tau_{a}}\left(c_{3}^{F}-c_{2}\right)$ Now left integral value of

truth membership indeterminacy and falsity membership functions of $\tilde{A}^{n}$ are

$V_{L_{T}}\left(\tilde{A}^{n}\right)=\int_{0}^{1} L_{T}^{-1}(h)=\frac{\left(2 w_{a}-1\right) a_{1}^{T}+a_{2}}{2 w_{a}}$ and $V_{L_{l}}\left(\tilde{A}^{n}\right)=\int_{0}^{1} L_{I}^{-1}(h)=\frac{\left(2 \eta_{a}-1\right) b_{1}^{v}+b_{2}}{2 \tau_{a}}$ and

$V_{L_{F}}\left(\tilde{A}^{n}\right)=\int_{0}^{1} L_{I}^{-1}(h)=\frac{\left(2 \tau_{a}-1\right) c_{1}^{\nu}+c_{2}}{2 \tau_{a}}$ respectively

And right integral value of truth, indeterminacy and falsity membership functions are $V_{R_{T}}\left(\tilde{A}^{n}\right)=\int_{0}^{1} R_{T}^{-1}(h)=\frac{\left(2 w_{a}-1\right) a_{3}^{T}+a_{2}}{2 w_{a}}, V_{R_{I}}\left(\tilde{A}^{n}\right)=\int_{0}^{1} R_{I}^{-1}(h)=\frac{\left(2 \eta_{a}+1\right) b_{3}^{I}-b_{2}}{2 \eta_{a}}$ and $V_{R_{F}}\left(\tilde{A}^{n}\right)=\int_{0}^{1} R_{F}^{-1}(h)=\frac{\left(2 \tau_{a}+1\right) c_{3}^{F}-c_{2}}{2 \tau_{a}}$ respectively.

The total integral value of the truth membership functions is

$V_{T}^{\alpha}\left(\tilde{A}^{n}\right)=\frac{\left(2 w_{a}-1\right) a_{3}^{T}+a_{2}}{2 w_{a}} \alpha+(1-\alpha) \frac{\left(2 w_{a}-1\right) a_{1}^{T}+a_{2}}{2 w_{a}}=\frac{a_{2}+\left(2 w_{a}-1\right)\left\{\alpha a_{3}^{T}+(1-\alpha) a_{1}^{T}\right\}}{2 w_{a}} ; \alpha \in[0,1]$

The total integral value of indeterminacy membership functions is

$V_{I}^{\beta}\left(\tilde{A}^{n}\right)=\frac{\left(2 \eta_{a}+1\right) b_{3}^{I}-b_{2}}{2 \eta_{a}} \beta+(1-\beta) \frac{\left(2 \eta_{a}-1\right) b_{1}^{v}+b_{2}}{2 \eta_{a}}=\frac{(1-2 \beta) b_{2}+b_{3}^{I}\left(2 \beta+2 \eta_{a}-1\right)}{2 \eta_{a}} ; \beta \in[0,1]$

The total integral value of falsity membership functions is

$V_{F}^{\gamma}\left(\tilde{A}^{n}\right)=\frac{\left(2 \tau_{a}+1\right) c_{3}^{F}-c_{2}}{2 \tau_{a}} \gamma+(1-\gamma) \frac{\left(2 \tau_{a}-1\right) c_{1}^{v}+c_{2}}{2 \tau_{a}}=\frac{(1-2 \gamma) c_{2}+c_{3}^{I}\left(2 \gamma+2 \tau_{a}-1\right)}{2 \tau_{a}} ; \gamma \in[0,1]$ 
Let

$$
\tilde{A}^{n}=\left(\left(a_{1}^{T}, a_{2}, a_{3}^{T} ; w_{a}\right),\left(b_{1}^{I}, b_{2}, b_{3}^{I} ; \eta_{a}\right)\left(c_{1}^{F}, c_{2}, c_{3}^{F} ; \tau_{a}\right)\right)
$$

and

$\tilde{B}^{n}=\left(\left(e_{1}^{T}, e_{2}, e_{3}^{T} ; w_{b}\right),\left(f_{1}^{I}, f_{2}, f_{3}^{I} ; \eta_{b}\right)\left(g_{1}^{F}, g_{2}, g_{3}^{F} ; \tau_{b}\right)\right)$ be two generalized triangular neutrosophic number then the following conditions hold good

i) If $\quad V_{T}^{\alpha}\left(\tilde{A}^{n}\right)<V_{T}^{\alpha}\left(\tilde{B}^{n}\right), \quad V_{I}^{\beta}\left(\tilde{A}^{n}\right)<V_{I}^{\beta}\left(\tilde{B}^{n}\right)$, and $\quad I_{F}^{\gamma}\left(\tilde{A}^{n}\right)<I_{F}^{\gamma}\left(\tilde{B}^{n}\right)$ for $\alpha, \beta, \gamma \in[0,1]$ then $\tilde{A}^{n}<\tilde{B}^{n}$

ii) If $\quad V_{T}^{\alpha}\left(\tilde{A}^{n}\right)>V_{T}^{\alpha}\left(\tilde{B}^{n}\right), \quad V_{I}^{\beta}\left(\tilde{A}^{n}\right)>V_{I}^{\beta}\left(\tilde{B}^{n}\right)$, and $\quad I_{F}^{\gamma}\left(\tilde{A}^{n}\right)>I_{F}^{\gamma}\left(\tilde{B}^{n}\right)$ for $\alpha, \beta, \gamma \in[0,1]$ then $\tilde{A}^{n}>\tilde{B}^{n}$

iii) If $\quad V_{T}^{\alpha}\left(\tilde{A}^{n}\right)=V_{T}^{\alpha}\left(\tilde{B}^{n}\right), \quad V_{I}^{\beta}\left(\tilde{A}^{n}\right)=V_{I}^{\beta}\left(\tilde{B}^{n}\right)$, and $\quad I_{F}^{\gamma}\left(\tilde{A}^{n}\right)=I_{F}^{\gamma}\left(\tilde{B}^{n}\right)$ for $\alpha, \beta, \gamma \in[0,1]$ then $\tilde{A}^{n}=\tilde{B}^{n}$

\section{MATHEMATICAL ANALYSIS}

\subsection{Formulation of Neutrosophic Programming with imprecise coefficient}

A multi-objective neutrosophic non-linear programming problem with imprecise coefficient can be formulated as

Minimize $\tilde{f}_{k_{0}}(x)=\sum_{t=1}^{T_{k_{0}}} \xi_{k_{0} t} \tilde{c}_{k_{0} t} \prod_{j=1}^{n} x_{j}^{a_{k_{0} j}}$ for $k_{0}=1,2, \ldots ., p$

Such that $\tilde{f}_{i}(x)=\sum_{t=1}^{T_{i}} \xi_{i t} \tilde{c}_{i t} \prod_{j=1}^{n} x_{j}^{a_{i j}} \leq \xi_{i} \tilde{b}_{i}$ for $i=1,2, \ldots ., m$

$$
x_{j}>0 \quad j=1,2, \ldots, n
$$

Here $\xi_{k_{0}}, \xi_{i t}, \xi_{i}$ are the signum function used to indicate sign of term in the equation. $\tilde{c}_{k_{0} t}>0, \tilde{c}_{i t}>0 . a_{k_{0} t j}, a_{i t j}$ are real numbers for all $i, t, k_{0}, j$.

Here

$$
\begin{aligned}
& \tilde{c}_{k_{0} t}=\left(\left(c_{k_{0} t}^{1 T}, c_{k_{0} t}^{2}, c_{k_{0} t}^{3 T} ; w_{k_{0} t}\right)\left(c_{k_{0} t}^{1 I}, c_{k_{0} t}^{2}, c_{k_{0} t}^{3 I} ; \eta_{k_{0} t}\right)\left(c_{k_{0} t}^{1 F}, c_{k_{0} t}^{2}, c_{k_{0} t}^{3 F} ; \tau_{k_{0} t}\right)\right) ; \\
& \tilde{c}_{i t}=\left(\left(c_{i t}^{1 T}, c_{i t}^{2}, c_{i t}^{3 T} ; w_{i t}\right)\left(c_{i t}^{1 I}, c_{i t}^{2}, c_{i t}^{3 I} ; \eta_{i t}\right)\left(c_{i t}^{1 F}, c_{i t}^{2}, c_{i t}^{3 F} ; \tau_{i t}\right)\right) ; \\
& \tilde{b}_{i}=\left(\left(b_{i}^{1 T}, b_{i}^{2}, b_{i}^{3 T} ; w_{i}\right)\left(b_{i}^{1 I}, b_{i}^{2}, b_{i}^{3 I} ; \eta_{i}\right)\left(b_{i}^{1 F}, b_{i}^{2}, b_{i}^{3 F} ; \tau_{i}\right)\right) .
\end{aligned}
$$

Using total integral value of truth, indeterminacy and falsity membership functions we transform above Neutrosophic multi-objective programming with imprecise parameter as

$$
\begin{gathered}
\text { Minimize } \hat{f}_{1 k_{0}}(x ; \alpha)=\sum_{t=1}^{T_{k_{0}}} \xi_{k_{0} t} \hat{c}_{1 k_{0} t} \prod_{j=1}^{n} x_{j}^{a_{k 0 j}} \text { for } k_{0}=1,2, \ldots ., p \\
\text { Minimize } \hat{f}_{2 k_{0}}(x ; \beta)=\sum_{t=1}^{T_{k_{0}}} \xi_{k_{0} t} \hat{c}_{2 k_{0} t} \prod_{j=1}^{n} x_{j}^{a_{k 0 j}} \text { for } k_{0}=1,2, \ldots ., p
\end{gathered}
$$


Minimize $\hat{f}_{3 k_{0}}(x ; \gamma)=\sum_{t=1}^{T_{k_{0}}} \xi_{k_{0}} \hat{c}_{3 k_{0} t} \prod_{j=1}^{n} x_{j}^{a_{k 0 j}}$ for $k_{0}=1,2, \ldots ., p$

Such that $\hat{f}_{1 i}(x ; \alpha)=\sum_{t=1}^{T_{i}} \xi_{i t} \hat{c}_{1 i t} \prod_{j=1}^{n} x_{j}^{a_{i j}} \leq \xi_{i} \hat{b}_{1 i}$ for $i=1,2, \ldots, m$

$\hat{f}_{2 i}(x ; \beta)=\sum_{i=1}^{T_{i}} \xi_{i t} \hat{c}_{2 i t} \prod_{j=1}^{n} x_{j}^{a_{i j}} \leq \xi_{i} \hat{b}_{2 i}$ for $i=1,2, \ldots ., m$

$\hat{f}_{3 i}(x ; \gamma)=\sum_{t=1}^{T_{i}} \xi_{i t} \hat{c}_{3 i t} \prod_{j=1}^{n} x_{j}^{a_{i j}} \leq \xi_{i} \hat{b}_{3 i}$ for $i=1,2, \ldots ., m$

$x_{j}>0 ; \alpha, \beta, \gamma \in[0,1] j=1,2, \ldots ., n$

Here $\xi_{k_{0}}, \xi_{i t}, \xi_{i}$ are the signum function used to indicate sign of term in the equation. $\hat{c}_{1 k_{0} t}>0, \hat{c}_{1 i t}>0 ; \hat{b}_{1 i}>0$ denote the total integral value of truth membership function i.e $\quad \hat{c}_{1 k_{0} t}=\frac{c_{k_{0} t}^{2}+\left(2 w_{k_{0} t}-1\right)\left\{\alpha c_{k_{0} t}^{3 \mu}+(1-\alpha) c_{k_{0} t}^{1 \mu}\right\}}{2 w_{k_{0} t}}, \hat{c}_{1 i t}=\frac{c_{i t}^{2}+\left(2 w_{i t}-1\right)\left\{\alpha c_{i t}^{3 \mu}+(1-\alpha) c_{i t}^{1 \mu}\right\}}{2 w_{i t}}$ and $\hat{b}_{1 i}=\frac{b_{i}^{2}+\left(2 w_{i}-1\right)\left\{\alpha b_{i}^{3 \mu}+(1-\alpha) b_{i}^{1 \mu}\right\}}{2 w_{i}}$ and $\hat{c}_{2 k_{0} t}>0, \hat{c}_{2 i t}>0 ; \hat{b}_{2 i}>0$ denote the total integral value of indeterminacy-membership function i.e $\hat{c}_{2 k_{0} t}=\frac{2 \eta_{k_{0} t} c_{k_{0} t}^{3 I}+(1-2 \beta)\left\{c_{k_{0} t}^{2}-c_{k_{0} t}^{3 I}\right\}}{2 \eta_{k_{0} t}}, \hat{c}_{2 i t}=\frac{2 \eta_{i t} c_{i t}^{3 I}+(1-2 \beta)\left\{c_{i t}^{2}-c_{i t}^{3 I}\right\}}{2 \eta_{i t}}$, and $\hat{b}_{2 i}=\frac{2 \eta_{i} b_{i}^{3 I}+(1-2 \beta)\left\{b_{i}^{2}-b_{i}^{3 I}\right\}}{2 \eta_{i}}$. and $\hat{c}_{3 k_{0} t}>0, \hat{c}_{3 i t}>0 ; \hat{b}_{3 i}>0$ denote the total integral value of falsity-membership function i.e $\hat{c}_{3 k_{0} t}=\frac{2 \tau_{k_{0} t} c_{k_{0} t}^{3 F}+(1-2 \gamma)\left\{c_{k_{0} t}^{2}-c_{k_{0} t}^{3 F}\right\}}{2 \tau_{k_{0} t}}$, $\hat{c}_{3 i t}=\frac{2 \tau_{i t} c_{i t}^{3 F}+(1-2 \gamma)\left\{c_{i t}^{2}-c_{i t}^{3 F}\right\}}{2 \tau_{i t}}$, and $\hat{b}_{2 i}=\frac{2 \tau_{i} b_{i}^{3 F}+(1-2 \gamma)\left\{b_{i}^{2}-b_{i}^{3 F}\right\}}{2 \tau_{i}}$.

4.2. Neutrosophic Non-linear Programming (NNLP) Optimization to solve Parameterized Multi-objective Non-linear Programming Problem (PMONLP) A multi-objective non-linear parametric neutrosophic programming (MONNLP) Problem can be formulated as

Minimize $\left\{f_{1}(x ; \alpha), \ldots ., f_{p}(x ; \alpha), f_{p+1}(x ; \beta), \ldots ., f_{2 p}(x ; \beta), f_{2 p+1}(x ; \gamma), \ldots ., f_{3 p}(x ; \gamma)\right\}^{T}$

Subject to $g_{j}(x ; \alpha) \leq b_{j} ; \quad j=1,2, \ldots . ., m$

$$
\begin{gathered}
g_{j}(x ; \beta) \leq b_{j} ; \quad j=1,2, \ldots . ., m \\
g_{j}(x ; \gamma) \leq b_{j} ; \quad j=1,2, \ldots . ., m
\end{gathered}
$$




$$
x>0 \alpha, \beta, \gamma \in[0,1]
$$

Following Zimmermann [30],we have presented a solution algorithm to solve the MONLP Problem by fuzzy optimization technique.

Step-1: Solve the MONLP (2) as a single objective non-linear programming problem $p$ times by taking one of the objective at a time and ignoring the others.These solutions are known as ideal solutions. Let $x^{i}$ be the respective optimal solution for the $i^{\text {th }}$ different objectives with same constraints and evaluate each objective values for all these $i^{\text {th }}$ optimal solutions.

Step-2: From the result of step -1 determine the corresponding values for every objective for each derived solutions. With the values of all objectives at each ideal solutions ,pay-off matrix can be formulated as follows

$$
\begin{gathered}
f_{1}(x ; \alpha) \\
x^{1} \\
x^{2} \\
\vdots \\
x^{3 p}\left[\begin{array}{ccccccccc}
f_{1}^{*}\left(x^{1} ; \alpha\right) & \ldots & f_{p}^{*}\left(x^{1} ; \alpha\right) & f_{p+1}^{*}\left(x^{1} ; \beta\right) & \ldots & f_{2 p}^{*}\left(x^{1} ; \beta\right) & f_{2 p+1}^{*}\left(x^{1} ; \gamma\right) & \ldots & f_{3 p}^{*}\left(x^{1} ; \gamma\right) \\
f_{1}^{*}\left(x^{2} ; \alpha\right) & \ldots & f_{p}^{*}\left(x^{2} ; \alpha\right) & f_{p+1}^{*}\left(x^{2} ; \beta\right) & \ldots & f_{2 p}^{*}\left(x^{2} ; \beta\right) & f_{2 p+1}^{*}\left(x^{2} ; \gamma\right) & \ldots & f_{3 p}^{*}\left(x^{2} ; \gamma\right) \\
\ldots & \ldots & \ldots & \ldots & \ldots & \ldots & \ldots & \ldots & \ldots \\
f_{1}^{*}\left(x^{3 p} ; \alpha\right) & \ldots & f_{p}^{*}\left(x^{3 p} ; \alpha\right) & f_{p+1}^{*}\left(x^{3 p} ; \beta\right) & \ldots & f_{2 p}^{*}\left(x^{3 p} ; \beta\right) & f_{2 p+1}^{*}\left(x^{3 p} ; \gamma\right) & \ldots & f_{3 p}^{*}\left(x^{3 p} ; \gamma\right)
\end{array}\right]
\end{gathered}
$$

Here $x^{1}, x^{2}, \ldots \ldots, x^{3 p}$ are the ideal solution of the objectives $f_{1}(x ; \alpha), f_{2}(x ; \alpha), \ldots ., f_{p}(x ; \alpha), f_{p+1}(x ; \beta), f_{p+2}(x ; \beta), \ldots ., f_{2 p}(x ; \beta), f_{2 p+1}(x ; \gamma), f_{2 p+2}(x ; \gamma), \ldots ., f_{3 p}(x ; \gamma)$ respectively.

Step-3: For each objective $f_{k}(x ; \alpha), f_{k}(x ; \beta), f_{k}(x ; \gamma)$ find lower bound $L_{k}^{T}$ and the upper bound $U_{k}^{T}$

$$
\begin{aligned}
& U_{k}^{T}=\max \left\{f_{k}\left(x^{r^{*}} ; p^{*}\right)\right\} \text { and } \\
& L_{k}^{T}=\min \left\{f_{k}\left(x^{r^{*}} ; p^{*}\right)\right\} \text { where } p^{*}=\alpha, \beta, \gamma ; k=1,2, \ldots, 3 p
\end{aligned}
$$

For truth membership of objectives.

Step-4:We represent upper and lower bounds for indeterminacy and falsity membership of objectives as follows :

for $k=1,2, \ldots . .3 p$

$U_{k}^{F}=U_{k}^{T} \quad$ and $L_{k}^{F}=L_{k}^{T}+t\left(U_{k}^{T}-L_{k}^{T}\right)$;

$L_{k}^{I}=L_{k}^{T}$ and $U_{k}^{I}=L_{k}^{T}+s\left(U_{k}^{T}-L_{k}^{T}\right)$

Here $t, s$ are predetermined real numbers in $(0,1)$

Step-5: Define truth membership, indeterminacy membership and falsity membership functions as follows

for $k=1,2, \ldots \ldots . ., 3 p$ 
Truss Design Optimization with Imprecise Load and Stress in Neutrosophic En-vironment 455

$$
\begin{aligned}
& T_{k}\left(f_{k}(x)\right)=\left\{\begin{array}{cl}
1 & \text { if } f_{k}(x) \leq L_{k}^{T} \\
0 & \text { if } L_{k}^{T} \leq f_{k}(x) \leq U_{k}^{T} \\
& \text { if } f_{k}(x) \geq U_{k}^{T}
\end{array}\right. \\
& I_{k}\left(f_{k}(x)\right)=\left\{\begin{array}{cl}
1 & \text { if } f_{k}(x) \leq L_{k}^{I} \\
\exp \left\{\frac{U_{k}^{I}-f_{k}(x)}{U_{k}^{I}-L_{k}^{I}}\right\} & \text { if } L_{k}^{I} \leq f_{k}(x) \leq U_{k}^{I} \\
0 & \text { if } f_{k}(x) \geq U_{k}^{I}
\end{array}\right. \\
& F_{k}\left(f_{k}(x)\right)=\left\{\begin{array}{cc}
0 & \text { if } f_{k}(x) \leq L_{k}^{F} \\
\frac{1}{2}+\frac{1}{2} \tanh \left\{\left(f_{k}(x)-\frac{U_{k}^{F}+L_{k}^{F}}{2}\right) \theta_{k}\right\} & \text { if } L_{k}^{F} \leq f_{k}(x) \leq U_{k}^{F} \\
1 & \text { if } f_{k}(x) \geq U_{k}^{F}
\end{array}\right.
\end{aligned}
$$

Where $\theta_{k}=\frac{6}{U_{k}^{F}-L_{k}^{F}}, \psi=4$

Step-6:Now neutrosophic optimization method for MONLP problem with probabilistic operator gives an equivalent nonlinear programming problem as:

Maximize $\prod_{i=1}^{p}\left(T_{i}\left(f_{i}(x ; \alpha)\right)\right) \prod_{i=p+1}^{2 p}\left(T_{i}\left(f_{i}(x ; \beta)\right)\right) \prod_{i=2}^{3 p}\left(T_{i}\left(f_{i}(x ; \gamma)\right)\right)$

Miniimize $\prod_{i=1}^{p}\left(1-I_{i}\left(f_{i}(x ; \alpha)\right)\right) \prod_{i=p+1}^{2 p}\left(1-I_{i}\left(f_{i}(x ; \beta)\right)\right) \prod_{2 p+1}^{3 p}\left(1-I_{i}\left(f_{i}(x ; \gamma)\right)\right)$

Miniimize $\prod_{i=1}^{p}\left(1-F_{i}\left(f_{i}(x ; \alpha)\right)\right) \prod_{i=p+1}^{2 p}\left(1-F_{i}\left(f_{i}(x ; \beta)\right)\right) \prod_{2 p+1}^{3 p}\left(1-F_{i}\left(f_{i}(x ; \gamma)\right)\right)$

subject to

$0 \leq T_{i}\left(f_{i}(x ; \alpha)\right)+I_{i}\left(f_{i}(x ; \alpha)\right)+F_{i}\left(f_{i}(x ; \alpha)\right) \leq 3$;

$0 \leq T_{i}\left(f_{i}(x ; \beta)\right)+I_{i}\left(f_{i}(x ; \beta)\right)+F_{i}\left(f_{i}(x ; \beta)\right) \leq 3$;

$0 \leq T_{i}\left(f_{i}(x ; \gamma)\right)+I_{i}\left(f_{i}(x ; \gamma)\right)+F_{i}\left(f_{i}(x ; \gamma)\right) \leq 3$;

$g_{j}(x ; \alpha) \leq b_{j}$;

$g_{j}(x ; \beta) \leq b_{j} ;$

$g_{j}(x ; \gamma) \leq b_{j}$;

$x>0 \alpha, \beta, \gamma \in[0,1]$

$i=1,2, \ldots ., p ; j=1,2, \ldots ., m$

This crisp nonlinear programming problem can be solved by appropriate mathematical algorithm. 


\section{SOLUTION OF MULTI-OBJECTIVE STRUCTURAL OPTIMIZATION PROBLEM BY INTUITIONISTIC FUZZY OPTIMIZATION TECHNIQUE}

The multi-objective structural model (1)can be expressed as parametric neutrosophic form as

Minimize $W T(A ; \alpha)$; Minimize $W T(A ; \beta)$; Minimize $W T(A ; \gamma)$

Minimize $\delta(A ; \alpha)$;Minimize $\delta(A ; \beta)$; Minimize $\delta(A ; \gamma)$

subject to

$\sigma(A ; \alpha) \leq([\sigma] ; \alpha) ; \sigma(A ; \beta) \leq([\sigma] ; \beta) ; \sigma(A ; \gamma) \leq([\sigma] ; \gamma)$

$A^{\min } \leq A \leq A^{\max } \alpha, \beta, \gamma \in[0,1]$

Where $A=\left(A_{1}, A_{2}, \ldots, A_{n}\right)^{T}$

To solve the MOSOP (4) step 1 of 4.2 is used. After that according to step 2 pay-off matrix is formulated

\begin{tabular}{|c|c|c|c|c|c|}
\hline & $W T(A ; \alpha)$ & $V T(A ; \beta)$ & $W T(A ; \gamma)$ & $\delta(A ; \alpha)$ & $\delta(A ; \beta)$ \\
\hline$A^{1}$ & {$\left[W T^{*}\left(A^{1} ; \alpha\right)\right.$} & $W T^{*}\left(A^{1} ; \beta\right)$ & $W T^{*}\left(A^{1} ; \gamma\right)$ & $\delta^{*}\left(A^{1} ; \alpha\right)$ & $\delta^{*}\left(A^{1} ; \beta\right) \quad \delta^{*}\left(A^{1} ; \gamma\right)$ \\
\hline & $W T^{*}\left(A^{2} ; \alpha\right)$ & $W T^{*}\left(A^{2} ; \beta\right)$ & $W T^{*}\left(A^{2} ; \gamma\right)$ & $\delta^{*}\left(A^{2} ;\right.$ & $\delta^{*}\left(A^{2} ; \beta\right) \delta^{*}$ \\
\hline & $W T^{*}\left(A^{3} ; \alpha\right)$ & $W T^{*}\left(A^{3} ; \beta\right)$ & $W T^{*}\left(A^{3} ; \gamma\right)$ & $\delta^{*}\left(A^{3} ; \alpha\right)$ & $; \beta) \delta^{*}$ \\
\hline & & $\ldots$ & 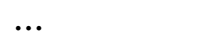 & 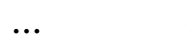 & ... \\
\hline & $W T^{*}\left(A^{6} ; \alpha\right)$ & $W T^{*}\left(A^{6} ; \beta\right)$ & $W T^{*}\left(A^{6} ; \gamma\right)$ & $\delta^{*}\left(A^{6} ; \alpha\right)$ & $O(A$ \\
\hline
\end{tabular}

Here $A^{1}, A^{2}, \ldots \ldots, A^{6}$ are the ideal solution of the objectives $W T(A ; \alpha), W T(A ; \beta), W T(A ; \gamma), \delta(A ; \alpha), \delta(A ; \beta), \delta(A ; \gamma)$ respectively.

For each objective $W T(A ; \alpha), W T(A ; \beta), W T(A ; \gamma), \delta(A ; \alpha), \delta(A ; \beta), \delta(A ; \gamma)$ find lower bound $L_{k}^{T}$ and the upper bound $U_{k}^{T}$ as

$U_{W T\left(A_{r}^{*} ; p\right)}^{T}=\max \left\{W T\left(A_{r}^{*} ; p\right)\right\} 0 \leq r \leq 6 ; p=\alpha, \beta, \gamma$ and

$L_{W T\left(A_{r}^{*} ; p\right)}^{T}=\min \left\{W T\left(A_{r}^{*} ; p\right)\right\} 0 \leq r \leq 6 ; p=\alpha, \beta, \gamma$

for truth membership of weight functions and

$U_{\delta\left(A_{r}^{*} ; p\right)}^{T}=\max \left\{\delta\left(A_{r}^{*} ; p\right)\right\} 0 \leq r \leq 6 ; p=\alpha, \beta, \gamma \quad$ and

$L_{\delta\left(A_{r}^{*} ; p\right)}^{T}=\min \left\{\delta\left(A_{r}^{*} ; p\right)\right\} 0 \leq r \leq 6 ; p=\alpha, \beta, \gamma$

for truth membership of deflection functions 
Similarly the upper and lower bounds for indeterminacy and falsity membership of weight objective function as

$$
\begin{aligned}
& U_{W T\left(A_{r}^{*} ; p\right)}^{F}=U_{W T\left(A_{r}^{*} ; p\right)}^{T} \text { and } L_{W T\left(A_{r}^{*} ; p\right)}^{F}=L_{W T\left(A_{r}^{*} ; p\right)}^{T}+t\left(U_{W T\left(A_{r}^{*} ; p\right)}^{T}-L_{W T\left(A_{r}^{*} ; p\right)}^{T}\right) ; \\
& L_{W T\left(A_{r}^{*} ; p\right)}^{I}=L_{W T\left(A_{r}^{*} ; p\right)}^{T} \text { and } U_{W T\left(A_{r}^{*} ; p\right)}^{I}=L_{W T\left(A_{r}^{*} ; p\right)}^{T}+s\left(U_{W T\left(A_{r}^{*} ; p\right)}^{T}-L_{W T\left(A_{r}^{*} ; p\right)}^{T}\right) ; 0 \leq r \leq 6 ; p=\alpha, \beta, \gamma
\end{aligned}
$$

And deflection objective function as

$$
\begin{aligned}
& U_{\delta\left(A_{r}^{*} ; p\right)}^{F}=U_{\delta\left(A_{r}^{*} ; p\right)}^{T} \text { and } L_{\delta\left(A_{r}^{*} ; p\right)}^{F}=L_{\delta\left(A_{r}^{*} ; p\right)}^{T}+t\left(U_{\delta\left(A_{r}^{*} ; p\right)}^{T}-L_{\delta\left(A_{r}^{*} ; p\right)}^{T}\right) ; \\
& L_{\delta\left(A_{r}^{*} ; p\right)}^{I}=L_{\delta\left(A_{r}^{*} ; p\right)}^{T} \text { and } U_{\delta\left(A_{r}^{*} ; p\right)}^{I}=L_{\delta\left(A_{r}^{*} ; p\right)}^{T}+s\left(U_{\delta\left(A_{r}^{*} ; p\right)}^{T}-L_{\delta\left(A_{r}^{*} ; p\right)}^{T}\right) ; 0 \leq r \leq 6 ; p=\alpha, \beta, \gamma
\end{aligned}
$$

Here $t, s$ are predetermined real numbers in $(0,1)$

Define truth membership, indeterminacy membership and falsity membership functions for weight and deflection as follows

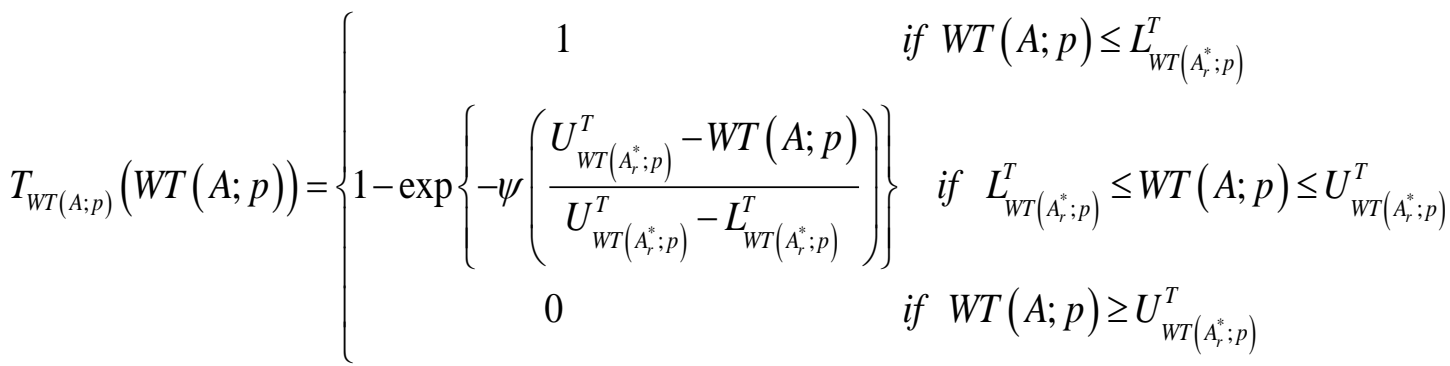

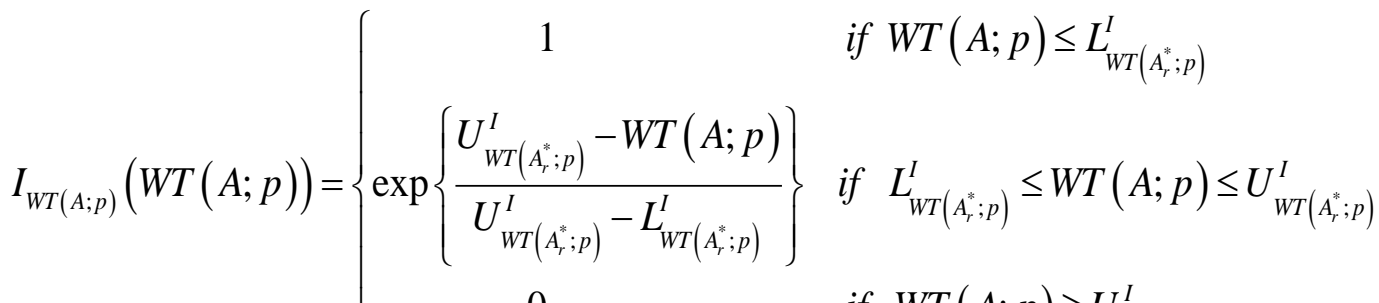

$$
\begin{aligned}
& 0 \quad \text { if } W T(A ; p) \geq U_{W T\left(A_{r}^{*} ; p\right)}^{I}
\end{aligned}
$$

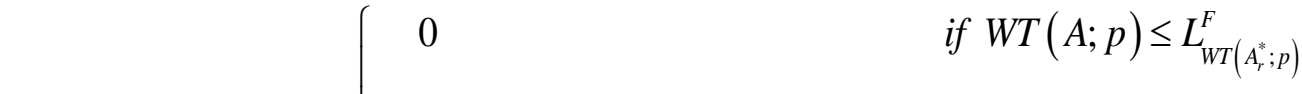

$$
\begin{aligned}
& F_{W T(A ; p)}(W T(A ; p))=\left\{\frac{1}{2}+\frac{1}{2} \tanh \left\{\left(W T(A ; p)-\frac{\left.U_{W T\left(A_{r}^{*} ; p\right)}^{F}+L_{W T\left(A_{r}^{*} ; p\right)}^{F}\right)}{2}\right) \theta_{W T\left(A_{r}^{*} ; p\right)}\right\}\right. \\
& \text { if } L_{W T\left(A_{r}^{*} ; p\right)}^{F} \leq W T(A ; p) \leq U_{W T\left(A_{r}^{*} ; p\right)}^{F} \\
& 1 \quad \text { if } W T(A ; p) \geq U_{W T\left(A_{r}^{*} ; p\right)}^{F}
\end{aligned}
$$




$$
\begin{aligned}
& \left(\begin{array}{cc}
1 & \text { if } \delta(A ; p) \leq L_{\delta\left(A_{r}^{*} ; p\right)}^{T}
\end{array}\right. \\
& T_{\delta(A ; p)}(\delta(A ; p))=\left\{1-\exp \left\{-\psi\left(\frac{U_{\delta\left(A_{r}^{*} ; p\right)}^{T}-\delta(A ; p)}{U_{\delta\left(A_{r}^{*} ; p\right)}^{T}-L_{\delta\left(A_{r}^{*} ; p\right)}^{T}}\right)\right\} \quad \text { if } \quad L_{\delta\left(A_{r}^{*} ; p\right)}^{T} \leq \delta(A ; p) \leq U_{\delta\left(A_{r}^{*} ; p\right)}^{T}\right. \\
& 0 \quad \text { if } \delta(A ; p) \geq U_{\delta\left(A_{r}^{*} ; p\right)}^{T}
\end{aligned}
$$

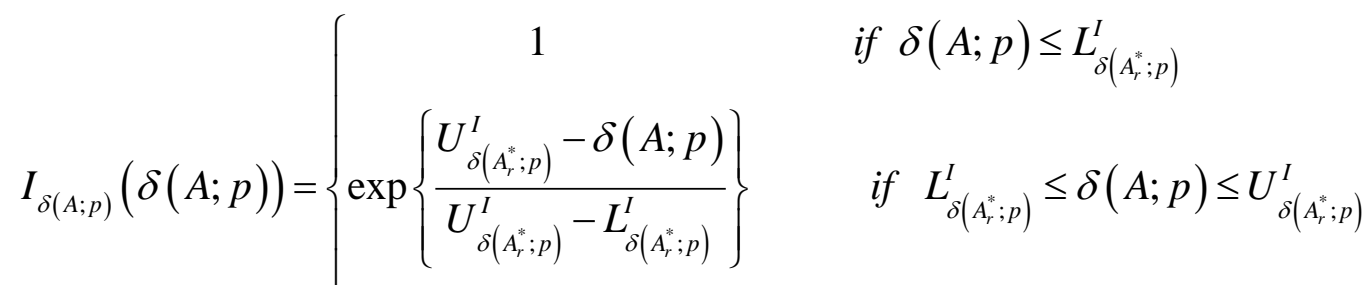

$$
\begin{aligned}
& 0 \quad \text { if } \delta(A ; p) \geq U_{\delta\left(A_{r}^{*} ; p\right)}^{I} \\
& \left(\begin{array}{cl}
0 & \text { if } \delta(A ; p) \leq L_{\delta\left(A_{r}^{*} ; p\right)}^{F}
\end{array}\right. \\
& F_{\delta(A ; p)}(\delta(A ; p))=\left\{\frac{1}{2}+\frac{1}{2} \tanh \left\{\left(\delta(A ; p)-\frac{\left.U_{\delta\left(A_{r}^{*} ; p\right)}^{F}+L_{\delta\left(A_{r}^{*} ; p\right)}^{F}\right)}{2}\right) \theta_{\delta\left(A_{r}^{*} ; p\right)}\right\}\right. \\
& \text { if } \quad L_{\delta\left(A_{r}^{*} ; p\right)}^{F} \leq \delta(A ; p) \leq U_{\delta\left(A_{r}^{*} ; p\right)}^{F} \\
& 1 \quad \text { if } \delta(A ; p) \geq U_{\delta\left(A_{r}^{*} ; p\right)}^{F}
\end{aligned}
$$

Where

$$
\theta_{W T\left(A_{r}^{*} ; p\right)}=\frac{6}{U_{W T\left(A_{r}^{*} ; p\right)}^{F}-L_{W T\left(A_{r}^{*} ; p\right)}^{F}} ; \theta_{\delta\left(A_{r}^{*} ; p\right)}=\frac{6}{U_{\delta\left(A_{r}^{*} ; p\right)}^{F}-L_{\delta\left(A_{r}^{*} ; p\right)}^{F}} ; \quad \psi=4
$$

$0 \leq r \leq 6 ; p=\alpha, \beta, \gamma$

Now neutrosophic optimization method for MONLP problem with probabilistic operator gives a equivalent nonlinear programming problem as:

$$
\text { Maximize }\left\{\begin{array}{l}
T_{W T(A ; \alpha)}(W T(A ; \alpha)) T_{W T(A ; \beta)}(W T(A ; \beta)) T_{W T(A ; \gamma)}(W T(A ; \gamma)) T_{\delta(A ; \alpha)}(\delta(A ; \alpha)) \\
T_{\delta(A ; \beta)}(\delta(A ; \beta)) T_{\delta(A ; \gamma)}(\delta(A ; \gamma))
\end{array}\right\}
$$

$$
\text { Miniimize }\left\{\begin{array}{l}
{\left[1-I_{W T(A ; \alpha)}(W T(A ; \alpha))\right]\left[1-I_{W T(A ; \beta)}(W T(A ; \beta))\right]\left[1-I_{W T(A ; \gamma)}(W T(A ; \gamma))\right]} \\
{\left[1-I_{\delta(A ; \alpha)}(\delta(A ; \alpha))\right]\left[1-I_{\delta(A ; \beta)}(\delta(A ; \beta))\right]\left[1-I_{\delta(A ; \gamma)}(\delta(A ; \gamma))\right]}
\end{array}\right\}
$$

Miniimize $\left\{\begin{array}{l}{\left[1-F_{W T(A ; \alpha)}(W T(A ; \alpha))\right]\left[1-F_{W T(A ; \beta)}(W T(A ; \beta))\right]\left[1-F_{W T(A ; \gamma)}(W T(A ; \gamma))\right]} \\ {\left[1-F_{\delta(A ; \alpha)}(\delta(A ; \alpha))\right]\left[1-F_{\delta(A ; \beta)}(\delta(A ; \beta))\right]\left[1-F_{\delta(A ; \gamma)}(\delta(A ; \gamma))\right]}\end{array}\right\}$ 
subject to

$0 \leq T_{W T(A ; p)}(W T(A ; p))+I_{W T(A ; p)}(W T(A ; p))+F_{W T(A ; p)}(W T(A ; p)) \leq 3$;

$0 \leq T_{\delta(A ; p)}(\delta(A ; p))+I_{\delta(A ; p)}(\delta(A ; p))+F_{\delta(A ; p)}(\delta(A ; p)) \leq 3$;

$\sigma(A) \leq[\sigma]$

$A^{\min } \leq A \leq A^{\max } 0 \leq r \leq 6 ; p=\alpha, \beta, \gamma ; \alpha, \beta, \gamma \in[0,1]$

This crisp nonlinear programming problem can be solved by appropriate mathematical algorithm.

\section{NUMERICAL ILLUSTRATION}

A well known three bar planer truss(Detailed formulation is given in appendix , Fig. 1) is considered to minimize weight of the structure $W T\left(A_{1}, A_{2}\right)$ and minimize the deflection $\delta\left(A_{1}, A_{2}\right)$ at a loading point of a statistically loaded three bar planer truss subject to stress constraints on each of the truss members

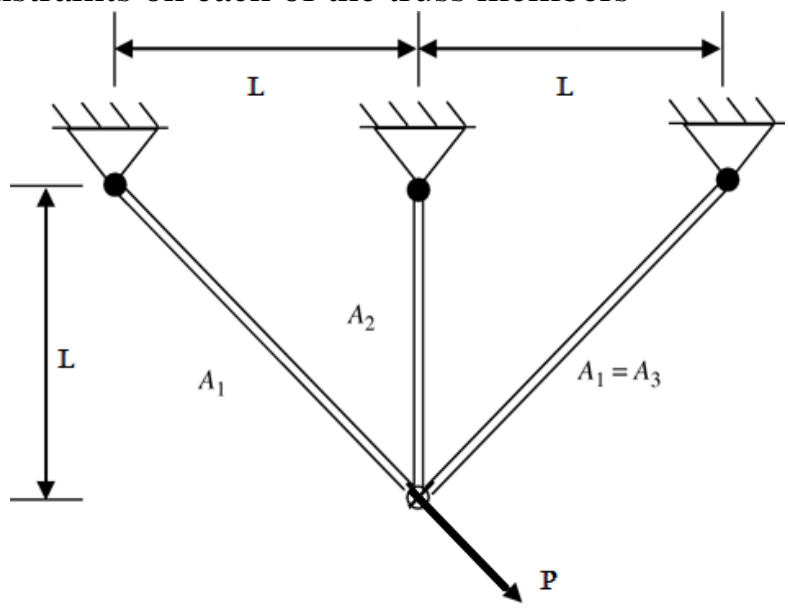

Fig 1: Design of the three-bar planar truss

The multi-objective optimization problem can be stated as follows

Minimize $W T\left(A_{1}, A_{2}\right)=\rho L\left(2 \sqrt{2} A_{1}+A_{2}\right)$

Minimize $\delta_{u}\left(A_{1}, A_{2}\right)=\frac{L P}{E A_{1}}$

Minimize $\delta_{v}\left(A_{1}, A_{2}\right)=\frac{L P}{E\left(A_{1}+\sqrt{2} A_{2}\right)}$

such that

$\sigma_{1}\left(A_{1}, A_{2}\right)=\frac{P\left(2 A_{1}+\sqrt{2} A_{2}\right)}{\left(A_{1}^{2}+\sqrt{2} A_{1} A_{2}\right)} \leq\left[\sigma_{1}^{T}\right]$ 


$$
\begin{aligned}
& \sigma_{2}\left(A_{1}, A_{2}\right)=\frac{P}{\left(A_{1}+\sqrt{2} A_{2}\right)} \leq\left[\sigma_{2}^{T}\right] \\
& \sigma_{3}\left(A_{1}, A_{2}\right)=\frac{P A_{2}}{\left(\sqrt{2} A_{1}^{2}+2 A_{1} A_{2}\right)} \leq\left[\sigma_{3}^{C}\right] \\
& A_{i}^{\min } \leq A_{i} \leq A_{i}^{\max } i=1,2 .
\end{aligned}
$$

Where applied load $\tilde{P}^{n} \equiv 2 \tilde{0}^{n} \equiv\left(\left(19,20,21 ; w_{p}\right)\left(18,20,22 ; \eta_{p}\right)\left(17,20,23 ; \tau_{p}\right)\right)$; material density $\rho=100 \mathrm{KN} / \mathrm{m}^{3}$; length $L=1 \mathrm{~m}$; Young's modulus $E=2 \times 10^{8} ; A_{1}=$ Cross section of bar-1 and bar-3; $A_{2}=$ Cross section of bar- $2 ; \delta_{u}$ and $\delta_{v}$ are the deflection of loaded joint along $u$ and $v$ axes respectively. $\left[\tilde{\sigma}_{1}^{T n}\right] \equiv 2 \tilde{0}^{n} \equiv\left(\left(19.5,20,20.5 ; w_{\sigma_{1}^{T}}\right)\left(18,20,21 ; \eta_{\sigma_{1}^{T}}\right)\left(17.5,20,21.5 \tau_{\sigma_{1}^{T}}\right)\right)$ and $\left[\tilde{\sigma}_{2}^{T n}\right] \equiv 2 \tilde{0}^{n} \equiv\left(\left(18.5,20,21 ; w_{\sigma_{2}^{T}}\right)\left(18,20,21.5 ; \eta_{\sigma_{2}^{T}}\right)\left(17.5,20,22 ; \tau_{\sigma_{2}^{T}}\right)\right)$ are $\quad$ maximum allowable tensile stress for bar 1 and bar 2 respectively, $\left[\tilde{\sigma}_{3}^{C n}\right] \equiv 1 \tilde{5}^{n} \equiv\left(\left(14,15,16 ; w_{\sigma_{3}^{c}}\right)\left(13,15,17 ; \eta_{\sigma_{3}^{c}}\right)\left(13.5,15,17.5 ; \tau_{\sigma_{3}^{c}}\right)\right)$ is $\quad$ maximum allowable compressive stress for bar 3 where $w_{p}=0.8, w_{\sigma_{1}^{T}}=0.7, w_{\sigma_{2}^{T}}=0.6, w_{\sigma_{3}^{c}}=0.9$ are degree of aspiration level of applied load, tensile stresses and compressive stress respectively and $\eta_{p}=0.4, \eta_{\sigma_{1}^{T}}=0.5, \eta_{\sigma_{2}^{T}}=0.3, \eta_{\sigma_{3}^{c}}=0.4$ $\tau_{p}=0.2, \tau_{\sigma_{1}^{T}}=0.2, \tau_{\sigma_{2}^{T}}=0.2, \tau_{\sigma_{3}^{C}}=0.1$ are degree of hesitancy and desperation level of applied load, tensile stresses and compressive stress respectively.

Now total integral value of membership and non-membership function are $\hat{P}_{1}=22.43+0.75 \alpha ; \hat{P}_{2}=19.5+5 \beta ; \hat{P}_{2}=15.5+15 \gamma ;$ $\hat{\sigma}_{11}^{T}=19.57+0.57 \alpha ; \hat{\sigma}_{21}^{T}=20+2 \beta ; \hat{\sigma}_{31}^{T}=17.75+7.5 \gamma ;$ $\hat{\sigma}_{12}^{T}=19.75+.42 \alpha ; \hat{\sigma}_{22}^{T}=20.25+3.75 \beta ; \hat{\sigma}_{32}^{T}=17+10 \gamma ;$ $\hat{\sigma}_{13}^{C}=14.55+0.89 \alpha ; \hat{\sigma}_{23}^{C}=14.5+5 \beta ; \hat{\sigma}_{33}^{C}=5+25 \gamma ;$

Using total integral values of coefficients, problem (6) can be transformed into Minimize $W T\left(A_{1}, A_{2}\right)=100\left(2 \sqrt{2} A_{1}+A_{2}\right)$

Minimize $\delta_{u}\left(A_{1}, A_{2} ; \alpha\right)=\frac{(22.43+0.75 \alpha)}{2 \times 10^{8} A_{1}}$

Minimize $\delta_{u}\left(A_{1}, A_{2} ; \beta\right)=\frac{(19.5+5 \beta)}{2 \times 10^{8} A_{1}}$

Minimize $\delta_{u}\left(A_{1}, A_{2} ; \gamma\right)=\frac{(15.5+15 \gamma)}{2 \times 10^{8} A_{1}}$ 
Truss Design Optimization with Imprecise Load and Stress in Neutrosophic En-vironment 461

Minimize $\delta_{v}\left(A_{1}, A_{2} ; \alpha\right)=\frac{(22.43+0.75 \alpha)}{\left(2 \times 10^{8}\right)\left(A_{1}+\sqrt{2} A_{2}\right)}$

Minimize $\delta_{v}\left(A_{1}, A_{2} ; \beta\right)=\frac{(19.5+5 \beta)}{\left(2 \times 10^{8}\right)\left(A_{1}+\sqrt{2} A_{2}\right)}$

Minimize $\delta_{v}\left(A_{1}, A_{2} ; \gamma\right)=\frac{(15.5+15 \gamma)}{\left(2 \times 10^{8}\right)\left(A_{1}+\sqrt{2} A_{2}\right)}$

such that

$$
\begin{aligned}
& \sigma_{11}\left(A_{1}, A_{2} ; \alpha\right) \equiv \frac{(22.43+0.75 \alpha)\left(2 A_{1}+\sqrt{2} A_{2}\right)}{\left(A_{1}^{2}+\sqrt{2} A_{1} A_{2}\right)} \leq 19.57+0.57 \alpha \\
& \sigma_{21}\left(A_{1}, A_{2} ; \beta\right) \equiv \frac{(19.5+5 \beta)\left(2 A_{1}+\sqrt{2} A_{2}\right)}{\left(A_{1}^{2}+\sqrt{2} A_{1} A_{2}\right)} \leq 20+2 \beta ; \\
& \sigma_{31}\left(A_{1}, A_{2} ; \gamma\right) \equiv \frac{(15.5+15 \gamma)\left(2 A_{1}+\sqrt{2} A_{2}\right)}{\left(A_{1}^{2}+\sqrt{2} A_{1} A_{2}\right)} \leq 15.5+15 \gamma ; \\
& \sigma_{12}\left(A_{1}, A_{2} ; \alpha\right) \equiv \frac{(22.43+0.75 \alpha)}{\left(A_{1}+\sqrt{2} A_{2}\right)} \leq 19.75+0.42 \alpha ; \\
& \sigma_{22}\left(A_{1}, A_{2} ; \beta\right) \equiv \frac{(19.5+5 \beta)}{\left(A_{1}+\sqrt{2} A_{2}\right)} \leq 20.25+3.75 \beta \\
& \sigma_{32}\left(A_{1}, A_{2} ; \gamma\right) \equiv \frac{(15.5+15 \gamma)}{\left(A_{1}+\sqrt{2} A_{2}\right)} \leq 17+10 \gamma ; \\
& \sigma_{13}\left(A_{1}, A_{2} ; \alpha\right) \equiv \frac{(22.43+0.75 \alpha) A_{2}}{\left(\sqrt{2} A_{1}^{2}+2 A_{1} A_{2}\right)} \leq 19.57+0.57 \alpha ; \\
& A_{i}^{\min } \leq A_{i} \leq A_{i}^{\max }(i=1,2 . \alpha, \beta, \gamma \in[0,1] \\
& \sigma_{23}\left(A_{1}, A_{2} ; \beta\right) \equiv \frac{(19.5+5 \beta) A_{2}}{\left(\sqrt{2} A_{1}^{2}+2 A_{1} A_{2}\right)} \leq 20+2 \beta \\
& \left(\sqrt{2} A_{1}^{2}+2 A_{1} A_{2}\right)
\end{aligned}
$$

According to step 2 pay-off matrix can be formulated as follows 


\begin{tabular}{|c|c|c|c|c|c|c|}
\hline$W T\left(A_{1}, A_{2}\right)$ & $\delta_{u}\left(A_{1}, A_{2} ; \alpha\right)$ & $\delta_{u}\left(A_{1}, A_{2} ; \beta\right)$ & $\delta_{u}\left(A_{1}, A_{2} ; \gamma\right)$ & $\delta_{v}\left(A_{1}, A_{2} ; \alpha\right)$ & $\delta_{v}\left(A_{1}, A_{2} ; \beta\right)$ & $\delta_{v}\left(A_{1}, A_{2} ; \gamma\right)$ \\
\hline$A^{1}[5.994110$ & 12.52100 & 12.23464 & 12.15401 & 7.229002 & 7.063671 & 7.07121 \\
\hline 17.16237 & 4.486000 & 4.128718 & 4.051560 & 2.419306 & 2.226623 & 2.185012 \\
\hline 15.95051 & 4.519218 & 3.90 & 4.051560 & 3.048520 & 2.580241 & 2.680513 \\
\hline 19.14214 & 4.519218 & 4.135986 & 3.10 & 1.908612 & 1.713182 & 1.284062 \\
\hline 19.14214 & 4.48600 & 4.128718 & 4.051560 & 1.858162 & 1.710171 & 1.678211 \\
\hline 19.14214 & 4.519218 & 3.90 & 4.051560 & 1.908612 & 1.615433 & 1.678211 \\
\hline 19.14214 & 4.519218 & 4.135986 & 3.10 & 1.908612 & 1.713182 & 1.284062 \\
\hline
\end{tabular}

Here

$$
\begin{aligned}
& U_{W T\left(A_{1}, A_{2}\right)}^{F}=U_{W T\left(A_{1}, A_{2}\right)}^{T}=19.14214, L_{W T\left(A_{1}, A_{2}\right)}^{F}=L_{W T\left(A_{1}, A_{2}\right)}^{T}+\varepsilon_{W T\left(A_{1}, A_{2}\right)}=5.994110+\varepsilon_{W T\left(A_{1}, A_{2}\right)} ; \\
& L_{W T\left(A_{1}, A_{2}\right)}^{I}=L_{W T\left(A_{1}, A_{2}\right)}^{T}=5.994110, U_{W T\left(A_{1}, A_{2}\right)}^{I}=L_{W T\left(A_{1}, A_{2}\right)}^{T}+\xi_{W T\left(A_{1}, A_{2}\right)}=5.994110+\xi_{W T\left(A_{1}, A_{2}\right)} \\
& \text { such that } 0<\varepsilon_{W T\left(A_{1}, A_{2}\right)}, \xi_{W T\left(A_{1}, A_{2}\right)}<(19.14214-5.994110) ; \\
& \psi=4, \theta_{W T\left(A_{1}, A_{2}\right)}=\frac{6}{U_{W T\left(A_{1}, A_{2}\right)}^{F}-L_{W T\left(A_{1}, A_{2}\right)}^{F}}, \theta_{W T\left(A_{1}, A_{2}\right)}=\frac{6}{U_{W T\left(A_{1}, A_{2}\right)}^{F}-L_{W T\left(A_{1}, A_{2}\right)}^{F}} \\
& U_{\delta_{u}\left(A_{1}, A_{2} ; \alpha\right)}^{F}=U_{\delta_{u}\left(A_{1}, A_{2} ; \alpha\right)}^{T}=12.52100, L_{\delta_{u}\left(A_{1}, A_{2} ; \alpha\right)}^{F}=L_{\delta_{u}\left(A_{1}, A_{2} ; \alpha\right)}^{T}+\varepsilon_{\delta_{u}\left(A_{1}, A_{2} ; \alpha\right)}=4.486000+\varepsilon_{\delta_{u}\left(A_{1}, A_{2} ; \alpha\right)} ; \\
& L_{\delta_{u}\left(A_{1}, A_{2} ; \alpha\right)}^{I}=L_{\delta_{u}\left(A_{1}, A_{2} ; \alpha\right)}^{T}=4.486000, U_{\delta_{u}\left(A_{1}, A_{2} ; \alpha\right)}^{I}=L_{\delta_{u}\left(A_{1}, A_{2} ; \alpha\right)}^{T}+\xi_{\delta_{u}\left(A_{1}, A_{2} ; \alpha\right)}=4.486000+\xi_{\delta_{u}\left(A_{1}, A_{2} ; \alpha\right)}
\end{aligned}
$$

such that $0<\varepsilon_{\delta_{u}\left(A_{1}, A_{2} ; \alpha\right)}, \xi_{\delta_{u}\left(A_{1}, A_{2} ; \alpha\right)}<(12.52100-4.48600)$

$$
\begin{aligned}
& \psi=4, \theta_{\delta_{u}\left(A_{1}, A_{2} ; \alpha\right)}=\frac{6}{U_{\delta_{u}\left(A_{1}, A_{2} ; \alpha\right)}^{F}-L_{\delta_{u}\left(A_{1}, A_{2} ; \alpha\right)}^{F}}, \theta_{\delta_{u}\left(A_{1}, A_{2} ; \alpha\right)}=\frac{6}{U_{\delta_{u}\left(A_{1}, A_{2} ; \alpha\right)}^{F}-L_{\delta_{u}\left(A_{1}, A_{2} ; \alpha\right)}^{F}} \\
& U_{\delta_{u}\left(A_{1}, A_{2} ; \beta\right)}^{F}=U_{\delta_{u}\left(A_{1}, A_{2} ; \beta\right)}^{T}=12.23464, L_{\delta_{u}\left(A_{1}, A_{2} ; \beta\right)}^{F}=L_{\delta_{u}\left(A_{1}, A_{2} ; \beta\right)}^{T}+\varepsilon_{\delta_{u}\left(A_{1}, A_{2} ; \beta\right)}=3.90+\varepsilon_{\delta_{u}\left(A_{1}, A_{2} ; \beta\right)} \text {; } \\
& L_{\delta_{u}\left(A_{1}, A_{2} ; \beta\right)}^{I}=L_{\delta_{u}\left(A_{1}, A_{2} ; \beta\right)}^{T}=3.90, U_{\delta_{u}\left(A_{1}, A_{2} ; \beta\right)}^{I}=L_{\delta_{u}\left(A_{1}, A_{2} ; \beta\right)}^{T}+\xi_{\delta_{u}\left(A_{1}, A_{2} ; \beta\right)}=3.90+\xi_{\delta_{u}\left(A_{1}, A_{2} ; \beta\right)} \\
& \text { such that } 0<\varepsilon_{\delta_{u}\left(A_{1}, A_{2} ; \beta\right)}, \xi_{\delta_{u}\left(A_{1}, A_{2} ; \beta\right)}<(12.23464-3.90)
\end{aligned}
$$$$
\psi=4, \theta_{\delta_{u}\left(A_{1}, A_{2} ; \beta\right)}=\frac{6}{U_{\delta_{u}\left(A_{1}, A_{2} ; \beta\right)}^{F}-L_{\delta_{u}\left(A_{1}, A_{2} ; \beta\right)}^{F}}, \theta_{\delta_{u}\left(A_{1}, A_{2} ; \beta\right)}=\frac{6}{U_{\delta_{u}\left(A_{1}, A_{2} ; \beta\right)}^{F}-L_{\delta_{u}\left(A_{1}, A_{2} ; \beta\right)}^{F}}
$$$$
U_{\delta_{u}\left(A_{1}, A_{2} ; \gamma\right)}^{F}=U_{\delta_{u}\left(A_{1}, A_{2} ; \gamma\right)}^{T}=12.15401, L_{\delta_{u}\left(A_{1}, A_{2} ; \gamma\right)}^{F}=L_{\delta_{u}\left(A_{1}, A_{2} ; \gamma\right)}^{T}+\varepsilon_{\delta_{u}\left(A_{1}, A_{2} ; \gamma\right)}=3.1+\varepsilon_{\delta_{u}\left(A_{1}, A_{2} ; \gamma\right)} \text {; }
$$$$
L_{\delta_{u}\left(A_{1}, A_{2} ; \gamma\right)}^{I}=L_{\delta_{u}\left(A_{1}, A_{2} ; \gamma\right)}^{T}=3.10, U_{\delta_{u}\left(A_{1}, A_{2} ; \gamma\right)}^{I}=L_{\delta_{u}\left(A_{1}, A_{2} ; \gamma\right)}^{T}+\xi_{\delta_{u}\left(A_{1}, A_{2} ; \gamma\right)}=3.10+\xi_{\delta_{u}\left(A_{1}, A_{2} ; \gamma\right)}
$$

such that $0<\varepsilon_{\delta_{u}\left(A_{1}, A_{2} ; \gamma\right)}, \xi_{\delta_{u}\left(A_{1}, A_{2} ; \gamma\right)}<(12.15401-3.10)$

$$
\begin{aligned}
& \psi=4, \theta_{\delta_{u}\left(A_{1}, A_{2} ; \gamma\right)}=\frac{6}{U_{\delta_{u}\left(A_{1}, A_{2} ; \gamma\right)}^{F}-L_{\delta_{u}\left(A_{1}, A_{2} ; \gamma\right)}^{F}}, \theta_{\delta_{u}\left(A_{1}, A_{2} ; \gamma\right)}=\frac{6}{U_{\delta_{u}\left(A_{1}, A_{2} ; \gamma\right)}^{F}-L_{\delta_{u}}^{F}\left(A_{1}, A_{2} ; \gamma\right)} \\
& U_{\delta_{v}\left(A_{1}, A_{2} ; \alpha\right)}^{F}=U_{\delta_{v}\left(A_{1}, A_{2} ; \alpha\right)}^{T}=7.229002, L_{\delta_{v}\left(A_{1}, A_{2} ; \alpha\right)}^{F}=L_{\delta_{v}\left(A_{1}, A_{2} ; \alpha\right)}^{T}+\varepsilon_{\delta_{v}\left(A_{1}, A_{2} ; \alpha\right)}=1.858162+\varepsilon_{\delta_{v}\left(A_{1}, A_{2} ; \alpha\right)}
\end{aligned}
$$


Truss Design Optimization with Imprecise Load and Stress in Neutrosophic En-vironment 463

$L_{\delta_{v}\left(A_{1}, A_{2} ; \alpha\right)}^{I}=L_{\delta_{v}\left(A_{1}, A_{2} ; \alpha\right)}^{T}=1.858162, U_{\delta_{v}\left(A_{1}, A_{2} ; \alpha\right)}^{I}=L_{\delta_{v}\left(A_{1}, A_{2} ; \alpha\right)}^{T}+\xi_{\delta_{v}\left(A_{1}, A_{2} ; \alpha\right)}=1.858162+\xi_{\delta_{v}\left(A_{1}, A_{2} ; \alpha\right)}$ such that $0<\varepsilon_{\delta_{v}\left(A_{1}, A_{2} ; \alpha\right)}, \xi_{\delta_{v}\left(A_{1}, A_{2} ; \alpha\right)}<(7.229002-1.858162)$

$\psi=4, \theta_{\delta_{v}\left(A_{1}, A_{2} ; \alpha\right)}=\frac{6}{U_{\delta_{v}\left(A_{1}, A_{2} ; \alpha\right)}^{F}-L_{\delta_{v}\left(A_{1}, A_{2} ; \alpha\right)}^{F}}, \theta_{\delta_{v}\left(A_{1}, A_{2} ; \alpha\right)}=\frac{6}{U_{\delta_{v}\left(A_{1}, A_{2} ; \alpha\right)}^{F}-L_{\delta_{v}\left(A_{1}, A_{2} ; \alpha\right)}^{F}}$

$U_{\delta_{v}\left(A_{1}, A_{2} ; \beta\right)}^{F}=U_{\delta_{v}\left(A_{1}, A_{2} ; \beta\right)}^{T}=7.063671, L_{\delta_{v}\left(A_{1}, A_{2} ; \beta\right)}^{F}=L_{\delta_{v}\left(A_{1}, A_{2} ; \beta\right)}^{T}+\varepsilon_{\delta_{v}\left(A_{1}, A_{2} ; \beta\right)}=1.615433+\varepsilon_{\delta_{v}\left(A_{1}, A_{2} ; \beta\right)}$;

$L_{\delta_{v}\left(A_{1}, A_{2} ; \beta\right)}^{I}=L_{\delta_{v}\left(A_{1}, A_{2} ; \beta\right)}^{T}=1.615433, U_{\delta_{v}\left(A_{1}, A_{2} ; \beta\right)}^{I}=L_{\delta_{v}\left(A_{1}, A_{2} ; \beta\right)}^{T}+\xi_{\delta_{v}\left(A_{1}, A_{2} ; \beta\right)}=1.615433+\xi_{\delta_{v}\left(A_{1}, A_{2} ; \beta\right)}$

such that $0<\varepsilon_{\delta_{v}\left(A_{1}, A_{2} ; \beta\right)}, \xi_{\delta_{v}\left(A_{1}, A_{2} ; \beta\right)}<(7.063671-1.615433)$

$\psi=4, \theta_{\delta_{v}\left(A_{1}, A_{2} ; \beta\right)}=\frac{6}{U_{\delta_{v}\left(A_{1}, A_{2} ; \beta\right)}^{F}-L_{\delta_{v}\left(A_{1}, A_{2} ; \beta\right)}^{F}}, \theta_{\delta_{v}\left(A_{1}, A_{2} ; \beta\right)}=\frac{6}{U_{\delta_{v}\left(A_{1}, A_{2} ; \beta\right)}^{F}-L_{\delta_{v}\left(A_{1}, A_{2} ; \beta\right)}^{F}}$

$U_{\delta_{v}\left(A_{1}, A_{2} ; \gamma\right)}^{F}=U_{\delta_{v}\left(A_{1}, A_{2} ; \gamma\right)}^{T}=7.07121, L_{\delta_{v}\left(A_{1}, A_{2} ; \gamma\right)}^{F}=L_{\delta_{v}\left(A_{1}, A_{2} ; \gamma\right)}^{T}+\varepsilon_{\delta_{v}\left(A_{1}, A_{2} ; \gamma\right)}=1.284062+\varepsilon_{\delta_{v}\left(A_{1}, A_{2} ; \gamma\right)}$;

$L_{\delta_{v}\left(A_{1}, A_{2} ; \gamma\right)}^{I}=L_{\delta_{v}\left(A_{1}, A_{2} ; \gamma\right)}^{T}=1.284062, U_{\delta_{v}\left(A_{1}, A_{2} ; \gamma\right)}^{I}=L_{\delta_{v}\left(A_{1}, A_{2} ; \gamma\right)}^{T}+\xi_{\delta_{v}\left(A_{1}, A_{2} ; \gamma\right)}=1.284062+\xi_{\delta_{v}\left(A_{1}, A_{2} ; \gamma\right)}$

such that $0<\varepsilon_{\delta_{v}\left(A_{1}, A_{2} ; \gamma\right)}, \xi_{\delta_{v}\left(A_{1}, A_{2} ; \gamma\right)}<(7.07121-1.284062)$

$\psi=4, \theta_{\delta_{v}\left(A_{1}, A_{2} ; \gamma\right)}=\frac{6}{U_{\delta_{v}\left(A_{1}, A_{2} ; \gamma\right)}^{F}-L_{\delta_{v}\left(A_{1}, A_{2} ; \gamma\right)}^{F}}, \theta_{\delta_{v}\left(A_{1}, A_{2} ; \gamma\right)}=\frac{6}{U_{\delta_{v}\left(A_{1}, A_{2} ; \gamma\right)}^{F}-L_{\delta_{v}\left(A_{1}, A_{2} ; \gamma\right)}^{F}}$

Here nonlinear truth, indeterminacy and falsity membership function of objectives $W T\left(A_{1}, A_{2}\right) ; \delta_{u}\left(A_{1}, A_{2} ; \alpha\right) ; \delta_{u}\left(A_{1}, A_{2} ; \beta\right) ; \delta_{u}\left(A_{1}, A_{2} ; \gamma\right)$ and $\delta_{v}\left(A_{1}, A_{2} ; \alpha\right)$; $\delta_{v}\left(A_{1}, A_{2} ; \beta\right) ; \delta_{v}\left(A_{1}, A_{2} ; \gamma\right)$ are defined for $T=2$ as follows

$T_{W T\left(A_{1}, A_{2}\right)}\left(W T\left(A_{1}, A_{2}\right)\right)=\left\{\begin{array}{cc}1 & \text { if } W T\left(A_{1}, A_{2}\right) \leq 5.994110 \\ 1-\exp \left\{-4\left(\frac{19.14214-W T\left(A_{1}, A_{2}\right)}{19.14214-5.994110}\right)\right\} & \text { if } 5.994110 \leq W T\left(A_{1}, A_{2}\right) \leq 19.14214 \\ 0 & \text { if } W T\left(A_{1}, A_{2}\right) \geq 19.14214\end{array}\right.$ $I_{W T\left(A_{1}, A_{2}\right)}\left(W T\left(A_{1}, A_{2}\right)\right)=\left\{\begin{array}{c}1 \text { if } W T\left(A_{1}, A_{2}\right) \leq 5.994110+\xi_{W T\left(A_{1}, A_{2}\right)} \\ \exp \left\{\frac{19.14214-W T\left(A_{1}, A_{2}\right)}{19.14214-\left(5.994110+\xi_{W T\left(A_{1}, A_{2}\right)}\right)}\right\} \\ \text { if } 5.994110+\xi_{W T\left(A_{1}, A_{2}\right)} \leq W T\left(A_{1}, A_{2}\right) \leq 19.14214 \\ 0 \quad \text { if } W T\left(A_{1}, A_{2}\right) \geq 19.14214\end{array}\right.$ 


$$
\begin{aligned}
& \left(\begin{array}{cc}
0 & \text { if } W T\left(A_{1}, A_{2}\right) \leq 5.994110+\varepsilon_{W T\left(A_{1}, A_{2}\right)}
\end{array}\right. \\
& F_{W T\left(A_{1}, A_{2}\right)}\left(W T\left(A_{1}, A_{2}\right)\right)=\left\{\frac{1}{2}+\frac{1}{2} \tanh \left\{\left(W T\left(A_{1}, A_{2}\right)-\frac{19.14214+5.994110+\varepsilon_{W T\left(A_{1}, A_{2}\right)}}{2}\right) \theta_{W T\left(A_{1}, A_{2}\right)}\right\}\right. \\
& \text { if } 5.994110+\varepsilon_{W T\left(A_{1}, A_{2}\right)} \leq W T\left(A_{1}, A_{2}\right) \leq 19.14214 \\
& 1 \quad \text { if } W T\left(A_{1}, A_{2}\right) \geq 19.14214 \\
& T_{\delta_{u}\left(A_{1}, A_{2} ; \alpha\right)}\left(\delta_{u}\left(A_{1}, A_{2} ; \alpha\right)\right)=\left\{\begin{array}{cl}
1 & \text { if } \delta_{u}\left(A_{1}, A_{2} ; \alpha\right) \leq 4.48600 \\
1-\exp \left\{-4\left(\frac{12.52100-\delta_{u}\left(A_{1}, A_{2} ; \alpha\right)}{12.52100-4.48600}\right)\right\} & \text { if } 4.48600 \leq \delta_{u}\left(A_{1}, A_{2} ; \alpha\right) \leq 12.52100 \\
0 & \text { if } \delta_{u}\left(A_{1}, A_{2} ; \alpha\right) \geq 12.52100
\end{array}\right. \\
& I_{\delta_{u}\left(A_{1}, A_{2} ; \alpha\right)}\left(\delta_{u}\left(A_{1}, A_{2} ; \alpha\right)\right)=\left\{\begin{array}{c}
1 \quad \text { if } \delta_{u}\left(A_{1}, A_{2} ; \alpha\right) \leq 4.48600+\xi_{\delta_{u}\left(A_{1}, A_{2} ; \alpha\right)} \\
\exp \left\{\frac{12.52100-\delta_{u}\left(A_{1}, A_{2} ; \alpha\right)}{12.52100-\left(4.48600+\xi_{\delta_{u}\left(A_{1}, A_{2} ; \alpha\right)}\right)}\right\} \\
\text { if } 4.48600+\xi_{\delta_{u}\left(A_{1}, A_{2} ; \alpha\right)} \leq \delta_{u}\left(A_{1}, A_{2} ; \alpha\right) \leq 12.52100 \\
0 \quad \text { if } \delta_{u}\left(A_{1}, A_{2} ; \alpha\right) \geq 12.52100
\end{array}\right. \\
& F_{\delta_{u}\left(A_{1}, A_{2} ; \alpha\right)}\left(\delta_{u}\left(A_{1}, A_{2} ; \alpha\right)\right)=\left\{\begin{array}{cr}
0 & \text { if } \delta_{u}\left(A_{1}, A_{2} ; \alpha\right) \leq 4.48600+\varepsilon_{\delta_{u}\left(A_{1}, A_{2} ; \alpha\right.} \\
\frac{1}{2}+\frac{1}{2} \tanh \left\{\left(\delta_{u}\left(A_{1}, A_{2} ; \alpha\right)-\frac{\left.12.52100+4.48600+\varepsilon_{\delta_{u}\left(A_{1}, A_{2} ; \alpha\right)}\right)}{2}\right) \theta_{\delta_{u}\left(A_{1}, A_{2} ; \alpha\right)}\right\}
\end{array}\right. \\
& \text { if } 4.48600+\varepsilon_{\delta_{u}\left(A_{1}, A_{2} ; \alpha\right)} \leq \delta_{u}\left(A_{1}, A_{2} ; \alpha\right) \leq 12.52100 \\
& 1 \quad \text { if } \delta_{u}\left(A_{1}, A_{2} ; \alpha\right) \geq 12.52100
\end{aligned}
$$

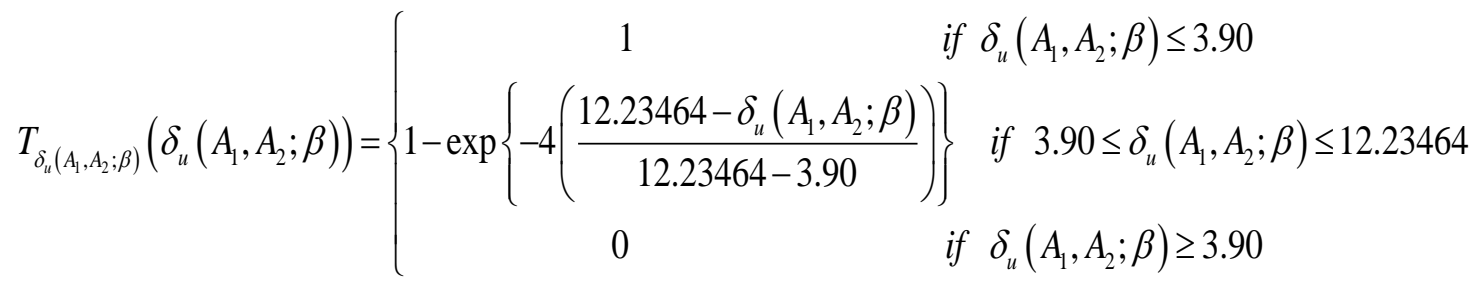


Truss Design Optimization with Imprecise Load and Stress in Neutrosophic En-vironment 465

$$
I_{\delta_{u}\left(A_{1}, A_{2} ; \beta\right)}\left(\delta_{u}\left(A_{1}, A_{2} ; \beta\right)\right)=\left\{\begin{array}{c}
1 \quad \text { if } \delta_{u}\left(A_{1}, A_{2} ; \beta\right) \leq 3.90+\xi_{\delta_{u}\left(A_{1}, A_{2} ; \beta\right)} \\
\exp \left\{\begin{array}{c}
12.23464-\delta_{u}\left(A_{1}, A_{2} ; \beta\right) \\
12.23464-\left(3.90+\xi_{\delta_{u}\left(A_{1}, A_{2} ; \beta\right)}\right)
\end{array}\right\} \\
0 \quad \text { if } 3.90+\xi_{\delta_{u}\left(A_{1}, A_{2} ; \beta\right)} \leq \delta_{u}\left(A_{1}, A_{2} ; \beta\right) \leq 12.23464 \\
0 \quad \text { if } \delta_{u}\left(A_{1}, A_{2} ; \beta\right) \geq 12.23464
\end{array}\right.
$$

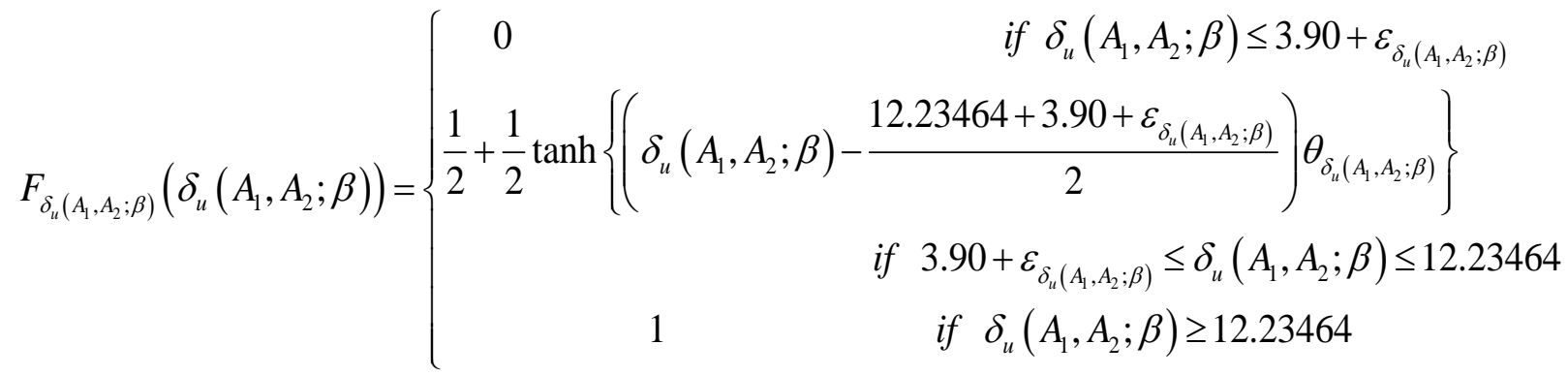

and

$$
T_{\delta_{u}\left(A_{1}, A_{2} ; \gamma\right)}\left(\delta_{u}\left(A_{1}, A_{2} ; \gamma\right)\right)=\left\{\begin{array}{cl}
1 & \text { if } \delta_{u}\left(A_{1}, A_{2} ; \gamma\right) \leq 3.10 \\
1-\exp \left\{-4\left(\frac{12.15401-\delta_{u}\left(A_{1}, A_{2} ; \gamma\right)}{12.15401-3.10}\right)\right\} & \text { if } 3.10 \leq \delta_{u}\left(A_{1}, A_{2} ; \gamma\right) \leq 12.15401 \\
0 & \text { if } \delta_{u}\left(A_{1}, A_{2} ; \gamma\right) \geq 3.10
\end{array}\right.
$$$$
I_{\delta_{u}\left(A_{1}, A_{2} ; \gamma\right)}\left(\delta_{u}\left(A_{1}, A_{2} ; \gamma\right)\right)=\left\{\begin{array}{c}
1 \quad \text { if } \delta_{u}\left(A_{1}, A_{2} ; \gamma\right) \leq 3.10+\xi_{\delta_{u}\left(A_{1}, A_{2} ; \gamma\right)} \\
\exp \left\{\begin{array}{c}
12.15401-\delta_{u}\left(A_{1}, A_{2} ; \gamma\right) \\
12.15401-\left(3.10+\xi_{\delta_{u}\left(A_{1}, A_{2} ; \gamma\right)}\right)
\end{array}\right\} \\
0 \quad \text { if } 3.10+\xi_{\delta_{u}\left(A_{1}, A_{2} ; \gamma\right)} \leq \delta_{u}\left(A_{1}, A_{2} ; \gamma\right) \leq 12.15401 \\
0 \quad \text { if } \delta_{u}\left(A_{1}, A_{2} ; \gamma\right) \geq 12.15401
\end{array}\right.
$$ 


$$
\begin{aligned}
& F_{\delta_{u}\left(A_{1}, A_{2} ; \gamma\right)}\left(\delta_{u}\left(A_{1}, A_{2} ; \gamma\right)\right)=\left\{\begin{array}{c}
0 \\
\text { if } \delta_{u}\left(A_{1}, A_{2} ; \gamma\right) \leq 3.10+\varepsilon_{\delta_{u}\left(A_{1}, A_{2} ; \gamma\right)} \\
\frac{1}{2}+\frac{1}{2} \tanh \left\{\left(\delta_{u}\left(A_{1}, A_{2} ; \gamma\right)-\frac{\left.12.15401+3.10+\varepsilon_{\delta_{u}\left(A_{1}, A_{2} ; \gamma\right)}\right)}{2}\right) \theta_{\delta_{u}\left(A_{1}, A_{2} ; \gamma\right)}\right\} \\
1 \quad \text { if } 3.10+\varepsilon_{\delta_{u}\left(A_{1}, A_{2} ; \gamma\right)} \leq \delta_{u}\left(A_{1}, A_{2} ; \gamma\right) \leq 12.15401 \\
\text { if } \delta_{u}\left(A_{1}, A_{2} ; \gamma\right) \geq 12.15401
\end{array}\right. \\
& T_{\delta_{v}\left(A_{1}, A_{2} ; \alpha\right)}\left(\delta_{v}\left(A_{1}, A_{2} ; \alpha\right)\right)=\left\{\begin{array}{cc}
1 & \text { if } \delta_{v}\left(A_{1}, A_{2} ; \alpha\right) \leq 1.858162 \\
1-\exp \left\{-4\left(\frac{7.229002-\delta_{v}\left(A_{1}, A_{2} ; \alpha\right)}{7.229002-1.858162}\right)\right\} & \text { if } 1.858162 \leq \delta_{v}\left(A_{1}, A_{2} ; \alpha\right) \leq 7.229002 \\
0 & \text { if } \delta_{v}\left(A_{1}, A_{2} ; \alpha\right) \geq 1.858162
\end{array}\right.
\end{aligned}
$$$$
I_{\delta_{v}\left(A_{1}, A_{2} ; \alpha\right)}\left(\delta_{v}\left(A_{1}, A_{2} ; \alpha\right)\right)=\left\{\begin{array}{c}
1 \text { if } \delta_{v}\left(A_{1}, A_{2} ; \alpha\right) \leq 1.858162+\xi_{\delta_{v}\left(A_{1}, A_{2} ; \alpha\right)} \\
\exp \left\{\frac{7.229002-\delta_{v}\left(A_{1}, A_{2} ; \alpha\right)}{7.229002-\left(1.858162+\xi_{\delta_{v}\left(A_{1}, A_{2} ; \alpha\right)}\right)}\right\} \\
\text { if } 1.858162+\xi_{\delta_{v}\left(A_{1}, A_{2} ; \alpha\right)} \leq \delta_{v}\left(A_{1}, A_{2} ; \alpha\right) \leq 7.229002 \\
0 \quad \text { if } \delta_{v}\left(A_{1}, A_{2} ; \alpha\right) \geq 7.229002
\end{array}\right\}
$$

$$
\begin{aligned}
& F_{\delta_{v}\left(A_{1}, A_{2} ; \alpha\right)}\left(\delta_{v}\left(A_{1}, A_{2} ; \alpha\right)\right)=\left\{\begin{array}{c}
0 \\
\frac{1}{2}+\frac{1}{2} \tanh \left\{\left(\delta_{v}\left(A_{1}, A_{2} ; \alpha\right)-\frac{7.229002+1.858162+\varepsilon_{\delta_{v}}\left(A_{1}, A_{2} ; \alpha\right)}{2}\right) \theta_{\delta_{v}\left(A_{1}, A_{2} ; \alpha\right)}\right\}
\end{array}\right. \\
& \text { if } 1.858162+\varepsilon_{\delta_{v}\left(A_{1}, A_{2} ; \alpha\right)} \leq \delta_{v}\left(A_{1}, A_{2} ; \alpha\right) \leq 7.229002 \\
& 1 \quad \text { if } \delta_{v}\left(A_{1}, A_{2} ; \alpha\right) \geq 7.229002 \\
& T_{\delta_{v}\left(A_{1}, A_{2} ; \beta\right)}\left(\delta_{v}\left(A_{1}, A_{2} ; \beta\right)\right)=\left\{\begin{array}{cl}
1 & \text { if } \delta_{v}\left(A_{1}, A_{2} ; \beta\right) \leq 1.615433 \\
1-\exp \left\{-4\left(\frac{7.063671-\delta_{v}\left(A_{1}, A_{2} ; \beta\right)}{7.063671-1.615433}\right)\right\} & \text { if } 1.615433 \leq \delta_{v}\left(A_{1}, A_{2} ; \beta\right) \leq 7.063671 \\
0 & \text { if } \delta_{v}\left(A_{1}, A_{2} ; \beta\right) \geq 1.615433
\end{array}\right.
\end{aligned}
$$


Truss Design Optimization with Imprecise Load and Stress in Neutrosophic En-vironment 467

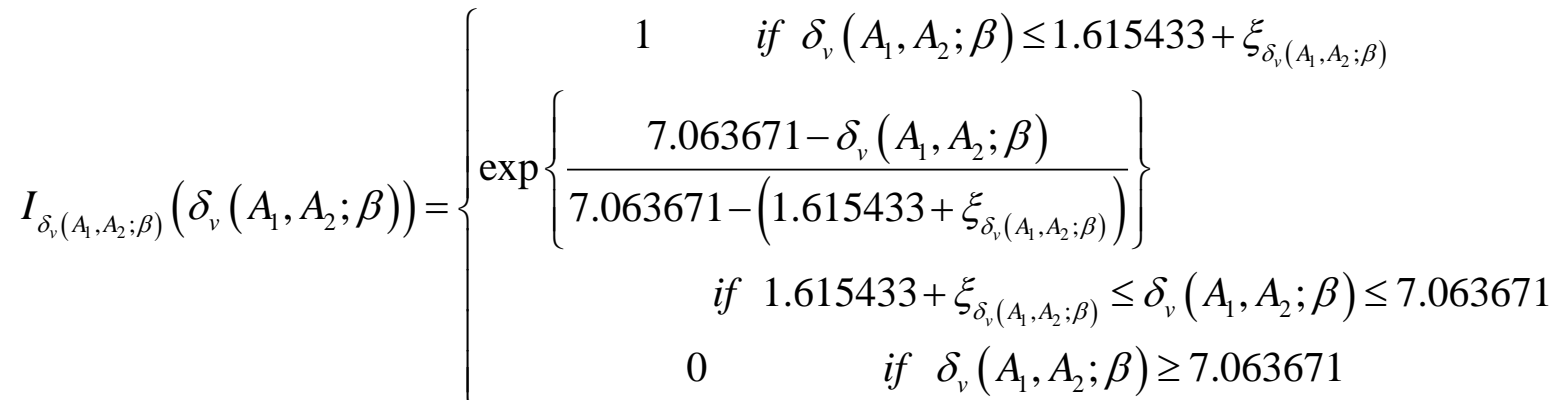

$$
\begin{aligned}
& \left(\begin{array}{cc}
0 & \text { if } \delta_{v}\left(A_{1}, A_{2} ; \beta\right) \leq 1.615433+\varepsilon_{\delta_{v}\left(A_{1}, A_{2} ; \beta\right)}
\end{array}\right. \\
& F_{\delta_{v}\left(A_{1}, A_{2} ; \beta\right)}\left(\delta_{v}\left(A_{1}, A_{2} ; \beta\right)\right)=\left\{\frac{1}{2}+\frac{1}{2} \tanh \left\{\left(\delta_{v}\left(A_{1}, A_{2} ; \beta\right)-\frac{7.063671+1.615433+\varepsilon_{\delta_{v}\left(A_{1}, A_{2} ; \beta\right)}}{2}\right) \theta_{\delta_{v}\left(A_{1}, A_{2} ; \beta\right)}\right\}\right. \\
& \text { if } 1.615433+\varepsilon_{\delta_{v}\left(A_{1}, A_{2} ; \beta\right)} \leq \delta_{v}\left(A_{1}, A_{2} ; \beta\right) \leq 7.063671 \\
& 1 \quad \text { if } \delta_{v}\left(A_{1}, A_{2} ; \beta\right) \geq 7.063671 \\
& T_{\delta_{v}\left(A_{1}, A_{2} ; \gamma\right)}\left(\delta_{v}\left(A_{1}, A_{2} ; \gamma\right)\right)=\left\{\begin{array}{cc}
1 & \text { if } \delta_{v}\left(A_{1}, A_{2} ; \gamma\right) \leq 1.284062 \\
1-\exp \left\{-4\left(\frac{7.07121-\delta_{v}\left(A_{1}, A_{2} ; \gamma\right)}{7.07121-1.284062}\right)\right\} & \text { if } 1.284062 \leq \delta_{v}\left(A_{1}, A_{2} ; \gamma\right) \leq 7.07121 \\
0 & \text { if } \quad \delta_{v}\left(A_{1}, A_{2} ; \gamma\right) \geq 1.284062
\end{array}\right. \\
& I_{\delta_{v}\left(A_{1}, A_{2} ; \gamma\right)}\left(\delta_{v}\left(A_{1}, A_{2} ; \gamma\right)\right)=\left\{\begin{array}{c}
1 \quad \text { if } \delta_{v}\left(A_{1}, A_{2} ; \gamma\right) \leq 1.284062+\xi_{\delta_{v}\left(A_{1}, A_{2} ; \gamma\right)} \\
\exp \left\{\frac{7.07121-\delta_{v}\left(A_{1}, A_{2} ; \gamma\right)}{7.07121-\left(1.284062+\xi_{\delta_{v}\left(A_{1}, A_{2} ; \gamma\right)}\right)}\right\} \\
\text { if } 1.284062+\xi_{\delta_{v}\left(A_{1}, A_{2} ; \gamma\right)} \leq \delta_{v}\left(A_{1}, A_{2} ; \gamma\right) \leq 7.07121
\end{array}\right. \\
& 0 \quad \text { if } \delta_{v}\left(A_{1}, A_{2} ; \gamma\right) \geq 7.07121
\end{aligned}
$$

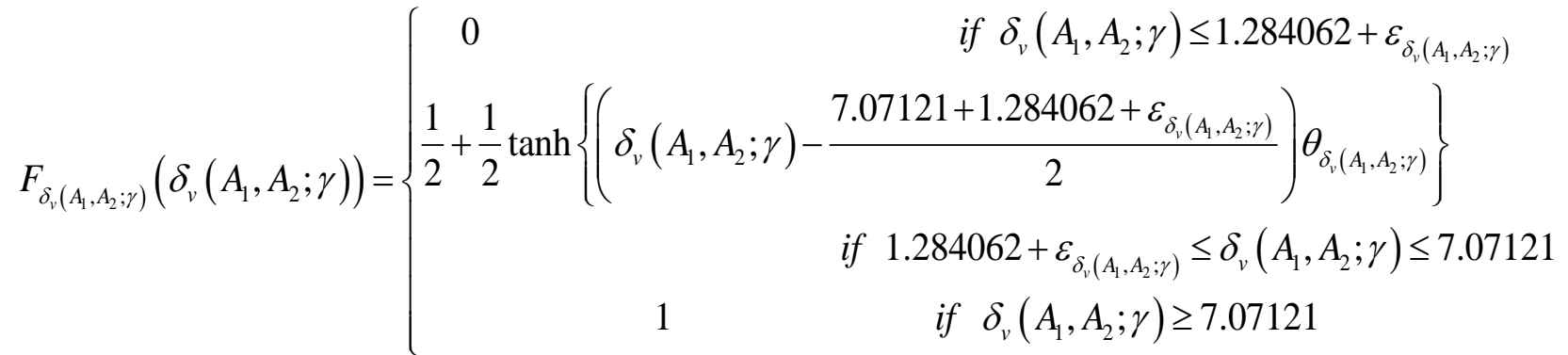

Using fuzzy, Intuitionistic, Neutrosophic Probabilistic Operator for truth; truth, falsity and truth, indeterminacy, falsity membership function respectively the optimal results of model (6) can be obtained and is given in table 2 . 
Table 2. Optimal weight and deflection for $\varepsilon_{W T\left(A_{1}, A_{2}\right)}, \xi_{W T\left(A_{1}, A_{2}\right)}=1.3$;

\begin{tabular}{|c|c|c|c|c|c|}
\hline Method & $A_{1}^{*} \times 10^{-4} m^{2}$ & $A_{2}^{*} \times 10^{-4} \mathrm{~m}^{2}$ & $W T^{*} \times 10^{2} K N$ & $\delta_{u}^{*} \times 10^{-7} \mathrm{~m}$ & $\delta_{v}^{*} \times 10^{-7} \mathrm{~m}$ \\
\hline $\begin{array}{l}\text { Fuzzy Max- } \\
\text { Min } \\
\text { Operator }\end{array}$ & 2.425445 & 1.568392 & 8.428587 & 0.5830738 & 0.3045585 \\
\hline $\begin{array}{l}\text { Fuzzy } \\
\text { Probabilistic } \\
\text { operator }\end{array}$ & 2.299305 & 4.006269 & 10.50968 & 8.698282 & 2.510978 \\
\hline $\begin{array}{l}\text { Intuitionistic } \\
\text { Probabilistic } \\
\text { Operator }\end{array}$ & 1.495007 & 2.604875 & 6.833394 & 13.37785 & 3.861852 \\
\hline $\begin{array}{l}\text { Neutrosophic } \\
\text { Probabilistic } \\
\text { Operator }\end{array}$ & 4.903401 & 4.807390 & 18.67630 & 4.078801 & 1.709098 \\
\hline
\end{tabular}

From the table 2.we may arrive to the conclusion that the weight is minimized when we have solved the model in intuitionistic optimization technique. As an explanation we can say in intuitionistic optimization we usually minimize non membership functions and maximize membership functions simultaneously. So it gives better result compare to fuzzy optimization where we only consider membership function for minimization. But as degree of acceptance is partially included in hesitancy and we minimize it in neutrosophic optimization it has given higher value than the result obtained in intuitionistic optimization.

\section{CONCLUSIONS}

In this paper, we have proposed a method to solve multi-objective structural model in neutrosophic environment. Here generalized neutrosophic number has been considered for applied load and stress parameter. The said model is solved by neutrosophic probabilistic operator and result is compared with fuzzy as well as intuitionistic probabilistic operator. The weight of the truss is more optimized by intuitionistic optimization technique. The main advantage of the described method is that it allows us to overcome the actual limitations in a problem where impreciseness of supplied data are involved during the specification of the objectives. This approximation method can be applied to optimize different models in various fields of engineering and sciences.

\section{ACKNOWLEDGEMENT:}

The research work of Mridula Sarkar is financed by Rajiv Gandhi National Fellowship (F1-17.1/2013-14-SC-wes-42549/(SA-III/Website)), Govt of India. 


\section{REFERENCES}

[1] D.M. Frangopol, R.B. Corotis, Reliability-based structural system optimization: state-of-the-art versus state-of-the-practice, in: F.Y. Cheng (Ed.), Proceeding of the 12th Conference on Analysis and Computation, Chicago, Analysis and Computation, ASCE, New York, 1996,67-78.

[2] M. Papadrakakis, N.D. Lagaros, Reliability-based structural optimization using neural networks and Monte Carlo simulation, Comput. Meth-ods Appl. Mech. Eng. 191(32) (2002) 3491-3507.

[3] A.Kaveh, M.Khayatazad, Ray optimization for size and shape optimization of truss structures. Computers and Structures ,117 (2013)82-94.

[4] M. Sonmez, Discrete optimum design of truss structures using artificial bee colony algorithm". Struct Multidiscip Optimiz, 43(1), (2011)85-97.

[5] R.E. Perez, K.Behdinan, Particle swarm approach for structural design optimization,Computers \& Structures, 85(19-20) (2007) 1579-1588.

[6] A.Kaveh, S.Talatahari , Particle swarm optimizer, ant colony strategy and harmony search scheme hybridized for optimization of truss structures. Computers and Structures. 87(56) (2009).267-283.

[7] G.C. Luh, C.Y.Lin, Optimal design of truss-structures using particle swarm optimization. Computers and Structures, 89(2324) (2011).2221-2232.

[8] A. Kaveh, H.Rahami, Nonlinear Analysis and Optimal Design of Structures via Force Method and Genetic Algorithm. Computers and Structures, 84, (2006) 770-778.

[9] Ali,Nicholas., Behdinan, Kumaran and Fawaz,Zouheir. Applicability and Viability of a GA based Finite Element Analysis Architecture for Structural Design Optimization. Computers and Structures, 81 (2003) 2259-2271.

[10] T.Dede, S.,L. Bekirog , Y.Ayvaz Weight minimization of trusses with genetic algorithm.Appl Soft Comput ,11(2) (2011)2565-2575.

[11] A.Kaveh, S. Motie, A. Mohammad, M.Moslehi, Magnetic charged system search: a new meta-heuristic algorithm for optimization. Acta Mech , 224, (2013)85-107.

[12] C. J. Shih, C. J. Chang, Mixed-discrete nonlinear fuzzy optimization for multiobjective engineering design. AIAA-94-1598-CP, (1994)2240-2246 .

[13] P. Hajela, C. J. Shih, Multi-objective optimum design in mixed integer and discrete design variable problems. AIAA Journal. 28(4) (1990) 670-675.

[14] D.Wang, W. H.Zhang, J. S .Jiang, "Truss Optimization on Shape and Sizing with Frequency Constraints", AIAA JOURNAL , 42(3) (2004).

[15] D.Wang, W. H.Zhang, J. S.Jiang, Truss shape optimization with multiple displacement constraints. Computer Methods in Applied Mechanics and Engineering. 191 (2002)3597-3612.

[16] P.Kripakaran, A.Gupta, and J.W.(Baugh Jr, A novel optimization approach for minimumcost design of trusses. Computers and Structures, 85(2007)1782179.

[17] L. A. Zadeh, Fuzzy set, Information and Control, 8(3) (1965)338-353,. 
[18] K. Atanassov, Intuitionistic fuzzy sets, Fuzzy sets and Systems, 20(1986)8796..

[19] S. Dey, and T.K.Roy, Optimized solution of two bar truss design using intuitionistic fuzzy optimization technique, International Journal of Information Engineering and Electronic Business,2014(3) (2014) 45-51.

[20] S .Dey, T.K.Roy, Multi-Objective Structural Optimization using Fuzzy and Intuitionistic Fuzzy Optimization Technique,I.J.Intelligent Systems and Applications,05 (2015)57-65.

[21] M. H. Shu, C. H. Cheng and J. R. Chang Using intuitionistic fuzzy sets for fault-tree analysis on printed circuit board assembly, Microelectronics Reliability, 46(12) (2006)2139-2148,.

[22] P. Grzegorzewski. Distances and orderings in a family of intuitionistic fuzzy numbers, EUSFLAT Conference, 223-227, 2003.

[23] H. B. Mitchell. Ranking-intuitionistic fuzzy numbers, International Journal of Uncertainty, Fuzziness and Knowledge-Based Systems, 12(3) (2004)377-386,

[24] G. Nayagam, V. Lakshmana, G. Venkateshwari and G. Sivaraman. Ranking of intuitionistic fuzzy numbers, IEEE International Conference on Fuzzy Systems,(IEEE World Congress on Computational Intelligence), (2008)19711974.

[25] H. M. Nehi. A new ranking method for intuitionistic fuzzy numbers, International Journal of Fuzzy Systems, 12(1) (2010)80-86,.

[26] S. Rezvani. Ranking method of trapezoidal intuitionistic fuzzy numbers, Annals of Fuzzy Mathematics and Informatics, 5(3) (2013)515-523,.

[27] D. F. Li. A ratio ranking method of triangular intuitionistic fuzzy numbers and its application to MADM problems, Computers and Mathematics with Applications, 60(6) (2010)1557-1570,

[28] F. Smarandache, Neutrosophy, neutrosophic probability, set and logic, Amer. Res. Press, Rehoboth, USA,105. (1998).

[29] H. Wang, F. Smarandache, Y. Q. Zhang, and R. Sunderraman, Single valued neutrosophic sets, Multispace and Multistructure, 4(2010)410-413,.

[30] Zimmermann, H.J.,1978, fuzzy linear programming with several objective function, Fuzzy sets and systems, 1, 46-55. 


\section{APPENDIX}

Consider the three-bar truss shown in fig-1. The bar have Young's modulus $E$ and the lengths are $l_{1}=l_{2}=l_{3}=L$. The design variables are cross-sectional areas $A_{1}, A_{2}$ and $A_{3}$ .But we assume that $A_{1}=A_{3}$. Weight of the structure is $W T=\rho l_{1} A_{1}+\rho l_{2} A_{2}+\rho l_{3} A_{3}=\rho L\left(2 \sqrt{2} A_{1}+A_{2}\right)$,where $\rho$ is the material density of each bar.The equilibrium equation is found by cutting out the free node as shown in fig1.The equilibrium equations in the direction $\mathrm{x}$ - and $\mathrm{y}$ - directions become in matrix form

$$
\left[\begin{array}{l}
P_{x} \\
P_{y}
\end{array}\right]=\left[\begin{array}{ccc}
\frac{1}{\sqrt{2}} & 0 & -\frac{1}{\sqrt{2}} \\
\frac{1}{\sqrt{2}} & 1 & \frac{1}{\sqrt{2}}
\end{array}\right]\left[\begin{array}{l}
N_{1} \\
N_{2} \\
N_{3}
\end{array}\right]
$$

i.e $F=B^{T} N$. Where $F$ represents the column matrix of external load, $N$ represents the column matrix of member's forces , $B^{T}$ represents the diagonal matrix of member of stiffness. We cannot obtain bar forces from equilibrium equations alone since the number of bars exceeds the number of degree-of-freedom.In order to find the bar forces ,or, rather, that appear in the constraints, we need to make use of Hook's law and geometry conditions. The extension of each bar corresponding to length and force are given in table 1 .

Table 1. Extension of Bars

\begin{tabular}{|c|c|c|c|}
\hline Bar & Length & Force & Extension \\
\hline Bar1 & $l_{1}=\sqrt{2} L$ & $N_{1}$ & $e_{1}=\frac{N_{1} \sqrt{2} L}{A_{1} E}$ \\
\hline Bar2 & $l_{2} L$ & $N_{2}$ & $e_{2}=\frac{N_{2} L}{A_{2} E}$ \\
\hline Bar3 & $l_{3}=\sqrt{2} L$ & $N_{3}$ & $e_{3}=\frac{N_{3} \sqrt{2} L}{A_{1} E}$ \\
\hline
\end{tabular}

we have $N_{i}=\frac{E A_{i} e_{i}}{l_{i}} i=1,2,3$

$\left[\begin{array}{l}N_{1} \\ N_{2} \\ N_{3}\end{array}\right]=\left[\begin{array}{c}\frac{e_{1} A_{1} E}{\sqrt{2} L} \\ \frac{e_{2} A_{2} E}{L} \\ \frac{e_{3} A_{1} E}{\sqrt{2} L}\end{array}\right]=\frac{E}{L}\left[\begin{array}{c}\frac{e_{1} A_{1}}{\sqrt{2}} \\ e_{2} A_{2} \\ \frac{e_{3} A_{3}}{\sqrt{2}}\end{array}\right],\left[\begin{array}{l}N_{1} \\ N_{2} \\ N_{3}\end{array}\right]=\frac{E}{L}\left[\begin{array}{ccc}\frac{A_{1}}{\sqrt{2}} & 0 & 0 \\ 0 & A_{2} & 0 \\ 0 & 0 & \frac{A_{1}}{\sqrt{2}}\end{array}\right]\left[\begin{array}{l}e_{1} \\ e_{2} \\ e_{3}\end{array}\right]$ 
We write these equations for all three bars in matrix form as $N=D e$, where $D=\frac{E}{L}\left[\begin{array}{ccc}\frac{A_{1}}{\sqrt{2}} & 0 & 0 \\ 0 & A_{2} & 0 \\ 0 & 0 & \frac{A_{1}}{\sqrt{2}}\end{array}\right], N=\left[\begin{array}{l}N_{1} \\ N_{2} \\ N_{3}\end{array}\right], e=\left[\begin{array}{l}e_{1} \\ e_{2} \\ e_{3}\end{array}\right]$

The compatibility equations relate the member displacement $e$ to the nodal displacement $\mathrm{r}$ by $e=B r$, the bar forces are obtained as $N=D B r$, s, The equilibrium equation $N_{i}=\frac{E A_{i} e_{i}}{l_{i}} i=1,2,3$ becomes $F=B^{T} N=B^{T} D B r=K r$ where $K=B^{T} D B$ is global stiffness matrix of the truss, which is

$$
\begin{aligned}
& K=\left[\begin{array}{ccc}
\frac{1}{\sqrt{2}} & 0 & -\frac{1}{\sqrt{2}} \\
\frac{1}{\sqrt{2}} & 1 & \frac{1}{\sqrt{2}}
\end{array}\right] \frac{E}{L}\left[\begin{array}{ccc}
\frac{A_{1}}{\sqrt{2}} & 0 & 0 \\
0 & A_{2} & 0 \\
0 & 0 & \frac{A_{1}}{\sqrt{2}}
\end{array}\right]\left[\begin{array}{ll}
\frac{1}{\sqrt{2}} & \frac{1}{\sqrt{2}} \\
0 & 1 \\
-\frac{1}{\sqrt{2}} & \frac{1}{\sqrt{2}}
\end{array}\right] \\
& =\frac{E}{L}\left[\begin{array}{ll}
\frac{A_{2}}{\sqrt{2}} & -\frac{A_{2}}{2} \\
-\frac{A_{2}}{2} & \frac{A_{1}}{\sqrt{2}}+A_{2}
\end{array}\right]
\end{aligned}
$$

Thus we obtain the displacement of free node as $r=K^{-1} F$

$$
\left[\begin{array}{l}
r_{1} \\
r_{2}
\end{array}\right]=\frac{2 L}{E A_{1}\left(A_{1}+\sqrt{2} A_{2}\right)}\left[\begin{array}{ll}
\frac{A_{1}}{\sqrt{2}}+A_{2} & 0 \\
0 & \frac{A_{1}}{2}
\end{array}\right]\left[\begin{array}{l}
P_{x} \\
P_{y}
\end{array}\right]=\left[\begin{array}{c}
\frac{\sqrt{2} L P_{x}}{E A_{1}} \\
\frac{\sqrt{2} L P_{y}}{E A_{1}\left(A_{1}+\sqrt{2} A_{2}\right)}
\end{array}\right]
$$

i.e the horizontal deflection of loaded joint is $r_{1}=\frac{\sqrt{2} L P_{x}}{E A_{1}}$

the vertical deflection of loaded joint is $r_{2}=\frac{\sqrt{2} L P_{y}}{E\left(A_{1}+\sqrt{2} A_{2}\right)}$

so stresses may be written as $\sigma=A N=A D B r$ where $A=\left[\begin{array}{ccc}\frac{1}{A_{1}} & 0 & 0 \\ 0 & \frac{1}{A_{2}} & 0 \\ 0 & 0 & \frac{1}{A_{1}}\end{array}\right]$ 
Truss Design Optimization with Imprecise Load and Stress in Neutrosophic En-vironment 473

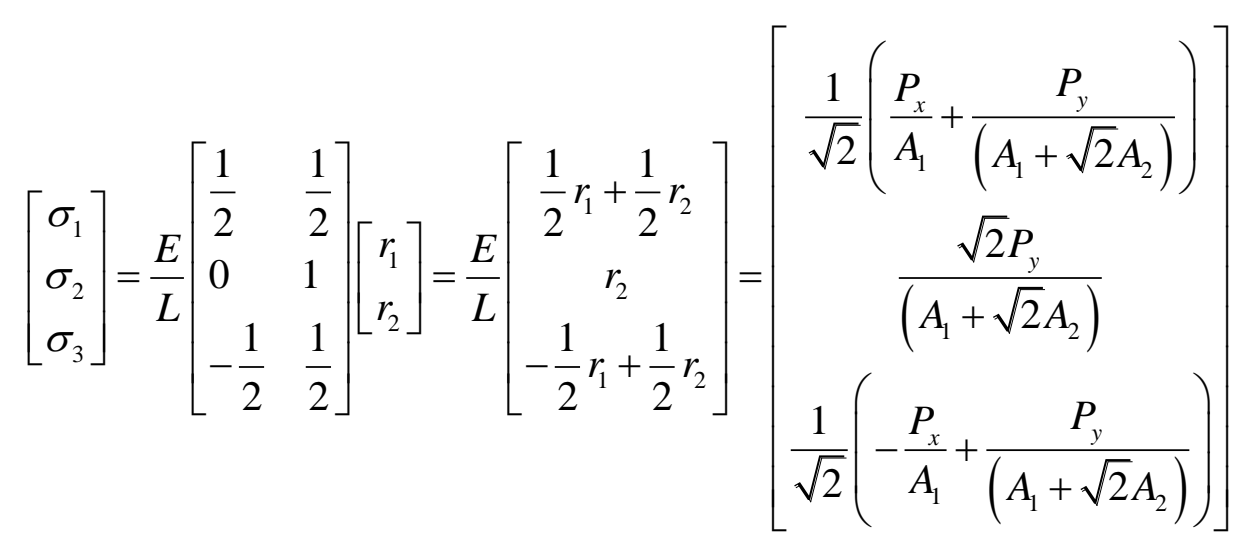

The tensile stress of bar 1 is $\sigma_{1} \equiv \frac{1}{\sqrt{2}}\left(\frac{P_{x}}{A_{1}}+\frac{P_{y}}{\left(A_{1}+\sqrt{2} A_{2}\right)}\right)$

The tensile stress of bar 2 is $\sigma_{2} \equiv \frac{\sqrt{2} P_{y}}{\left(A_{1}+\sqrt{2} A_{2}\right)}$

The compressive stress of bar 3 is $\sigma_{3} \equiv \frac{1}{\sqrt{2}}\left(-\frac{P_{x}}{A_{1}}+\frac{P_{y}}{\left(A_{1}+\sqrt{2} A_{2}\right)}\right)$

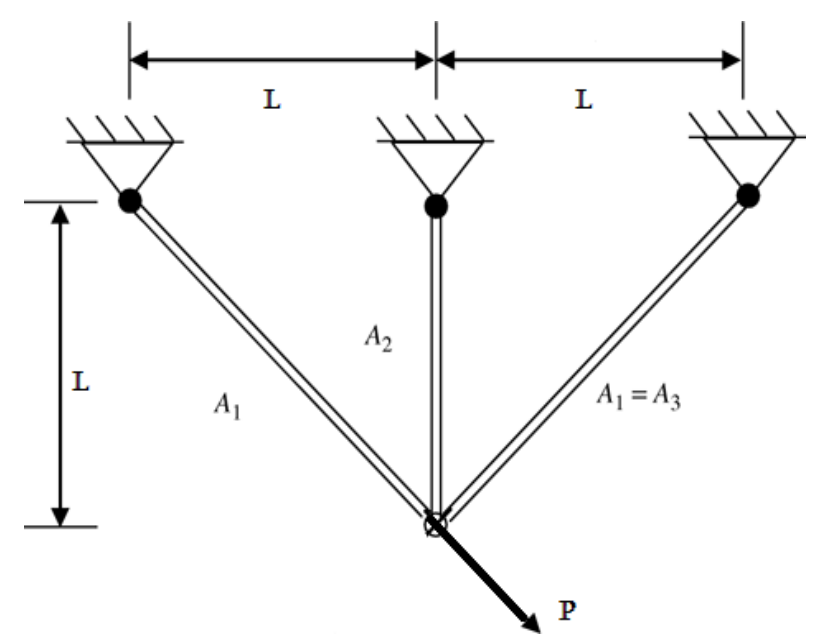

Fig .1. Design of three bar planar truss

Considering $P_{x}=P \cos \theta$ and $P_{y}=P \sin \theta$ assuming $\theta=45^{\circ}$ multi-objective structural design problem, the diagram of which is presented by Fig.1 can be formulated as

Minimize $W T\left(A_{1}, A_{2}\right)=\rho L\left(2 \sqrt{2} A_{1}+A_{2}\right)$ 
Minimize $\delta_{u}\left(A_{1}, A_{2}\right)=\frac{L P}{E A_{1}}$

Minimize $\delta_{v}\left(A_{1}, A_{2}\right)=\frac{L P}{E\left(A_{1}+\sqrt{2} A_{2}\right)}$

such that

$$
\begin{aligned}
& \sigma_{1}\left(A_{1}, A_{2}\right)=\frac{P\left(2 A_{1}+\sqrt{2} A_{2}\right)}{\left(A_{1}^{2}+\sqrt{2} A_{1} A_{2}\right)} \leq\left[\sigma_{1}^{T}\right] \\
& \sigma_{2}\left(A_{1}, A_{2}\right)=\frac{P}{\left(A_{1}+\sqrt{2} A_{2}\right)} \leq\left[\sigma_{2}^{T}\right] \\
& \sigma_{3}\left(A_{1}, A_{2}\right)=\frac{P A_{2}}{\left(\sqrt{2} A_{1}^{2}+2 A_{1} A_{2}\right)} \leq\left[\sigma_{3}^{C}\right] \\
& A_{i}^{\min } \leq A_{i} \leq A_{i}^{\max } i=1,2 .
\end{aligned}
$$

